\title{
1 New recoil transfer chamber for thermalization of heavy 2 ions produced in fusion-evaporation reactions
}

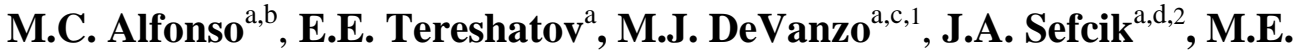 \\ Bennett $^{\mathrm{a}, 3}$, D.A. Mayorov ${ }^{\mathrm{a}, \mathrm{b}}$, T.A. Werke ${ }^{\mathrm{a}, \mathrm{b}}$, and C.M. Folden III ${ }^{\mathrm{a}, *}$ \\ ${ }^{a}$ Cyclotron Institute, Texas A\&M University, College Station, TX 77843 USA \\ ${ }^{\mathrm{b}}$ Department of Chemistry, Texas A\&M University, College Station, TX 77842 \\ USA \\ ${ }^{c}$ Department of Physics, Astronomy, and Geosciences, Towson University, \\ Towson, MD 21252 USA \\ ${ }^{\mathrm{d}}$ Department of Physics and Geosciences, Angelo State University, San Angelo, \\ TX 76909 USA \\ * Corresponding author. Email address: Folden@ comp.tamu.edu (C. M. Folden \\ III)
}

\begin{abstract}
A new Recoil Transfer Chamber (RTC) has been designed, fabricated, and characterized at the Cyclotron Institute at Texas A\&M University. The design is based on a gas stopper that was previously in routine use at the National Superconducting Cyclotron Laboratory. This new RTC uses He gas to stop ions, and a combination of a static electric field and gas flow to maximize the extraction efficiency. In offline experiments, $\mathrm{a}^{228} \mathrm{Th}$ source was used to produce ${ }^{216} \mathrm{Po}$ which was successfully extracted even though it has a short half-life. In online experiments using the products of the ${ }^{118} \mathrm{Sn}\left({ }^{40} \mathrm{Ar}, 6 \mathrm{n}\right){ }^{152} \mathrm{Er}$ reaction, an efficiency of several tens of percent was measured.
\end{abstract}

Keywords. Recoil transfer chamber, Gas stopper, Gas catcher, Heavy elements, Fusion-evaporation reactions

\footnotetext{
${ }^{1}$ Present address: Lockheed Martin Space Systems Company, 230 Mall Boulevard, King of Prussia, PA 19406 USA

${ }^{2}$ Present address: CivilCorp, LLC, 2825 Wilcrest Drive, Suite 460, Houston, TX 77042 USA

${ }^{3}$ Present address: Nuclear Engineering Division, Argonne National Laboratory, Argonne, IL 60439 USA
} 


\section{1. Introduction}

The development of efficient gas stoppers, also known as gas catchers, gas cells, or recoil

3 transfer chambers (RTCs), has allowed a variety of sophisticated experiments utilizing

4 thermalized beams to be conducted. These systems can be installed at particle accelerator

5 facilities, where they give access to exotic nuclei far from the line of beta stability. Recent

6 experiments have included first chemistry studies of superheavy elements [1-4], precision mass

7 measurements using Penning traps [5-7], and delivery of radioactive beams to reacceleration

8 facilities $[8,9]$. These systems typically employ one of two designs. The first design consists of

9 a relatively simple single gas-filled chamber that uses the flow of the gas to extract ions [10-12].

10 The extraction efficiency of these systems is high, but the resulting beam of thermalized products

11 has a very large and poorly characterized emittance. This makes them unsuitable for next-

12 generation experiments, such as the transmission of superheavy ions to a mass spectrometer

13 (e.g., the FIONA project under development at Lawrence Berkeley National Laboratory). The

14 second design consists of many variations that all use radiofrequency (RF) oscillating electric

15 fields to guide the ions $[5,9,13-18]$. This reduces the extraction time and increases the

16 efficiency, but these RF systems are extremely complex (for example, [13, 14, 17-19]), and

17 sometimes require cryogenic cooling [15].

18 A new RTC has been developed that gives the efficient transport of ions using electric

19 fields without the complexity of an RF system. The new Texas A\&M RTC design is primarily

20 based on the gas stopper previously used at the National Superconducting Cyclotron Laboratory

21 (NSCL) [20-23]. (See Fig. 1 in [20] for a schematic). The RTC has been simulated, fabricated,

22 and characterized in both offline and online experiments. In the future, the RTC will be used to

23 perform "online" chemistry experiments at Texas A\&M University, where activity is produced

24 in an accelerator-based experiment and utilized within a few seconds. 


\section{2. Design}

In the NSCL gas stopper, the ions were slowed by a rotating variable angle degrader

3 (VAD) and a window, and stopped in a chamber filled with 750 torr (100 $\mathrm{kPa})$ of He. A

4 decreasing potential gradient created by a series of "ring" electrodes drew the ions toward a set

5 of four hemispherical, concentric "flower petal" electrodes (see Fig. 4.3 in [24] for a picture),

6 and these also had a potential gradient. This second gradient focused the ions toward an

7 extraction nozzle with a diameter of $0.6 \mathrm{~mm}$, where the flow of the gas carried the ions through

8 the nozzle [13, 25]. The NSCL gas stopper was in routine use but has now been

9 decommissioned. The new RTC incorporates the NSCL gas stopper's actual flower petal

10 assembly and includes design elements from the previous system.

11 A schematic of the new RTC design is shown in Fig. 1. In addition to the NSCL

12 components, the RTC incorporates a VAD, an entrance window, a He-filled stopping region,

13 ring electrodes, the flower petal electrodes, and a nozzle. The VAD is made of a single layer of

14 Mylar $^{\circledR}$ (polyethylene terephthalate), and the entrance window that separates the RTC gas from

15 the beamline vacuum is made of aluminized Mylar so that it can be biased (see the discussion in

16 Sec. 3). The window is supported by a metal grid with a "honeycomb" pattern, and the grid is

17 covered by a "protector screen" that is intended to prevent pieces of the window from being

18 drawn into the beamline if it fails. After passing through the window, the ions enter the "main

19 chamber" (MC) where they are stopped in He with pressure $P$. The He gas enters the MC from

20 an expansion chamber surrounding the $\mathrm{MC}$ by passing through the cylindrical "inner chamber

21 groove." Four ring electrodes transport the ions from the stopping region to the flower petal

22 focusing region. The ions are swept through the nozzle into an "aerosol chamber" (AC). In

23 future experiments, the ions will be attached to aerosol particles and be transported through a 24 capillary to a chemistry laboratory. The volume of the AC was kept small $\left(\sim 15 \mathrm{~cm}^{3}\right)$, since this 
1 reduces the flushing time [12]. Due to the small diameter of the nozzle $(0.6 \mathrm{~mm})$, a slight

2 difference in pressure (DP) can be maintained between the MC and the AC in order to ensure

3 that the gas flows the proper direction through the nozzle, while carrying the ions swiftly and

4 efficiently ( $\left.\mathrm{DP}=P_{\mathrm{MC}}-P_{\mathrm{AC}}\right)$. The downstream side of the $\mathrm{AC}$ is mounted on a bellows system

5 that allows it to be retracted; i.e., the three metal elements that form the downstream wall of the

6 AC in Fig. 1 can be withdrawn and the system operated without aerosols. All of the experiments

7 in the current work were performed with the downstream wall retracted. Two retractable $\mathrm{Si}$

8 detectors, the "MC detector" inserted through the inner chamber groove and the "AC detector"

9 inserted downstream of the nozzle, were used to measure the extraction efficiency.

\section{3. Simulations}

11 The design of the system was optimized through a series of simulations. The new RTC is

12 designed to stop product beams that have first been purified using physical pre-separation [26].

13 Specifically, the Momentum Achromat Recoil Spectrometer (MARS) [27, 28] was assumed to be

14 the physical pre-separator. MARS has been previously developed for the purification of a beam

15 of heavy element evaporation residues (EVRs) with energies of $150-250 \mathrm{keV} / \mathrm{u}$ [29]. The

16 nuclides ${ }^{85} \mathrm{Zr}$, ${ }^{169} \mathrm{Hf}$, and ${ }^{257} \mathrm{Rf}$ from group 4 of the Periodic Table were considered as model

17 heavy ions for the simulations, due to their importance in previous online chemistry experiments

18 and differing kinematics [30-33]. The expected energy and spatial distributions of ions exiting

19 MARS were simulated for the reactions ${ }^{70} \mathrm{Ge}\left({ }^{18} \mathrm{O}, 3 \mathrm{n}\right){ }^{85} \mathrm{Zr} ;{ }^{124} \mathrm{Sn}\left({ }^{50} \mathrm{Ti}, 5 \mathrm{n}\right){ }^{169} \mathrm{Hf}$; and

$20{ }^{208} \mathrm{~Pb}\left({ }^{50} \mathrm{Ti}, \mathrm{n}\right){ }^{257} \mathrm{Rf}$ using the LISE++ [34] and SRIM-2010 programs [35, 36], and the calculated

21 spatiokinematic properties are given in Table 1. The initial EVR energies exiting the target are

22 tens of $\mathrm{MeV}$, but they must be degraded to a few $\mathrm{MeV}$ for proper stopping in the gas. If the 
1 VAD is rotated to an angle $\theta$, then the total effective degrader thickness $d_{\text {total }}$ is a function of the nominal window thickness $d_{\text {window }}$ and the nominal VAD thickness $d_{\mathrm{VAD}}$ :

$$
d_{\text {total }}=d_{\text {window }}+d_{\mathrm{VAD}} /(\cos \theta) .
$$

4 The use of the rotating degrader gives maximum flexibility during an online experiment in case

5 the ions' range is much smaller or greater than predicted. The two Mylar degrader thicknesses,

6 the angle, and the He pressure were varied until the centroid of the stopped distribution was $\sim 2.5$

$7 \mathrm{~cm}$ beyond the window (in the center of the stopping region). The optimum angle was estimated

8 to be $\theta=20^{\circ}-30^{\circ}$ based on previously reported results [21-23], and calculations showed that the

9 optimum He pressure was $P=228$ torr $(30.4 \mathrm{kPa})$. Table 1 also gives the EVR energies that are

10 necessary for the ions to have the proper range at this pressure.

11 The SIMION ${ }^{\circledR}$ program [37] was used to simulate the trajectory of the thermalized ions due

12 to the electric field using the previously calculated stopped distributions as input. Factors such

13 as the size of the window opening, the distance to the first ring, the number of rings, the diameter

14 of the rings, the diameter of the flower petal electrodes, and the number of flower petal

15 electrodes were examined. The effects of large numbers of collisions between the ions and gas

16 atoms were simulated using the Statistical Diffusion Model user program for SIMION [38]. This

17 program requires the reduced mobilities of the ions, which were estimated using data from [39]

18 and are given in Table 1. Charge exchange was not considered due to a lack of reliable cross

19 section data; therefore, all ions were assumed to always be in the $1+$ charge state. The effects of

20 space charge were not considered since the expected rate of incoming ions and their energies are

21 both very low. As a result of the simulations, the Mylar window was chosen to be aluminized so

22 that it can be biased, which helps prevent the ions from moving toward the window. The

23 optimum number of rings was four; fewer rings resulted in decreased focusing of ions into the 
1 nozzle, while more rings did not increase the efficiency. The optimum potential gradient was

2 qualitatively similar to the one used at NSCL (see Fig. 2 in [23]), which consisted of a gentle

3 decrease in potential from the window through the rings and a steeper gradient from the first

4 flower petal to the nozzle. The steeper gradient caused the spatial distribution to compress in 5 preparation for extraction through the nozzle.

6 The flow of gas in the RTC was studied using the STAR-CCM $+{ }^{\circledR}$ program [40]. The total

7 flow rate, DP, and the size of the expansion chamber were varied systematically to minimize

8 eddies in the gas and ensure a smooth flow. The final calculated gas flow in the MC is shown in

9 Fig. 2(a), and a detail of the gas flow in the nozzle region is shown in Fig. 2(b). The calculated

10 mass flow rate through the nozzle increased with DP and was typically several $\mathrm{mg} / \mathrm{s}$

11 (corresponding to a few $\mathrm{L} / \mathrm{min}$ ). The $\mathrm{DP}$ between the $\mathrm{MC}$ and the $\mathrm{AC}$ was found to be critical,

12 since the gas flow carries the ions through the nozzle where no field is present.

13 A final series of simulations was conducted that merged the gas flow results from STAR-

14 CCM+ with the ion trajectory calculation capabilities of SIMION. The results validated the design

15 principles established in Sec. 2. For each of the three reactions given in Table 1, conditions were

16 found that gave a high probability of reaching the nozzle. (Simulations in the nozzle itself

17 should be interpreted cautiously due to the very non-equilibrium conditions found there as the

18 gas accelerates to high velocities while rapidly compressing and then expanding). The final

19 design is shown in Fig. 3. The ions enter from the left of the figure and are intended to stop

20 within the first $5 \mathrm{~cm}$ of the MC. The biased window $(6 \mathrm{~cm}$ diameter) and inner chamber (shown

21 in yellow) focus the ions into the ring electrodes (shown in green). The rings help to ensure that

22 the front focusing (stopping) region does not interfere with the flower petal focusing region. The

23 flower petals (shown in purple, dark blue, yellow, and light blue) focus the ions into the nozzle 
1 (shown in red). The ions enter the AC chamber (shown in yellow) and will travel through tubes

2 (shown in red) that transport gas to and from the AC. The total distance from the window to the

3 nozzle is $13 \mathrm{~cm}$. The system uses a $45.7 \mathrm{~cm}$ flange to mount to MARS slightly downstream of

4 its focal plane. This design was fabricated, and the remainder of this manuscript discusses the 5 equipment and its experimental characterization.

\section{4. Gas-Handling System}

The purity of the He in the MC is important for the transmission of ions through the RTC, since the ions can react with any contaminants and possibly form neutral compounds or heavy

9 molecular ions [41, 42]. This decreases the effectiveness of the electric field; therefore, efforts

10 were made to significantly reduce the presence of hydrocarbon and other contaminants in the

11 system. He gas with an initial purity of $99.999 \%$ is first passed through a MonoTorr ${ }^{\circledR}$ Rare Gas

12 Purifier from SAES Pure Gas that reduces the concentration of common atmospheric gases to

$13 \mathrm{ppb}$ levels. All vacuum seals are made of $\mathrm{Cu}$ gaskets except the O-ring that seals the window,

14 which is made of Viton ${ }^{\circledR}$. The insulators are made of Macor ${ }^{\circledR}$ ceramic and polyphenylene sulfide

15 plastic; both of these materials have a low adsorption of moisture and low outgas rates to prevent

16 the release of contaminants. Typically, the entire chamber and gas handling system is baked at

$1790^{\circ} \mathrm{C}$ for $48 \mathrm{~h}$ before online experiments.

The gas-handling system is shown, schematically, in Fig. 4 and is controlled by a custom

19 interface designed in LABVIEW ${ }^{\circledR}$ [43]. The capillary connects the exit port of the AC to an oil-

20 free scroll pump with a nominal pump speed of $600 \mathrm{~L} / \mathrm{min}$. The flow rate is monitored and

21 controlled at the beginning of the capillary (downstream of the AC). The difference in pressure

22 between the MC and the AC chambers is controlled by independent all-metal control valves. 
1 Pressure in the MC is measured by a pressure transducer and DP is measured by a differential

2 capacitance manometer between the corresponding inlet lines.

\section{Preparatory Experiments}

As a proof-of-principle of RTC operation, the performance of the RTC was measured in a series of offline tests using a ${ }^{228} \mathrm{Th}$ source. Gaseous ${ }^{220} \mathrm{Rn}$ emanating from the source is neutral before decay [44], but its alpha daughter ${ }^{216} \mathrm{Po}$ (half-life $145 \pm 2 \mathrm{~ms}$ [45]) becomes charged upon production [46] and was used to simulate EVRs. (Errors in this manuscript are reported at the $1 \sigma$ confidence interval). The Mylar window was replaced with a metal disk and a $2.5 \mathrm{~cm}$ metal cylinder was mounted to the disk to support the source. The AC detector was a Canberra largearea $\left(\sim 2,000 \mathrm{~mm}^{2}\right)$, bakeable $\mathrm{Si}$ detector capable of operating at high pressures. The effects of voltage distribution, DP, and capillary inner diameter were studied by measuring the rate of ${ }^{216} \mathrm{Po}$ alpha decays on the AC detector, and an example energy spectrum is shown in Fig. 5. The optimized voltage distribution is given in the seventh column of Table 2 (starting with $420 \mathrm{~V}$ ), and the corresponding electric field is shown in Fig. 6(a). The yield increased as DP was increased from 0 to 10 torr $(2.0 \mathrm{kPa})$, but was constant above 10 torr. Either disabling the electric field or reducing the flow rate to zero resulted in a near-complete loss of extraction efficiency. These data show that the electric field is critical for carrying the short-lived ions through the main body of the MC, while the DP between the MC and the AC is critical for carrying the ions through the nozzle. The system was not capable of measuring the transportation time directly. The successful detection of heavy ions in the AC demonstrates that the fundamental design principles established in Sec. 2 have been satisfied.

The ${ }^{228} \mathrm{Th}$ source was also moved within the MC along the central axis and off the central axis in order to measure the influence of position on the yield of ${ }^{216} \mathrm{Po}$. The results are shown in 
1 Fig. 7. The yield was a strong function of position, and the relative efficiency when the source is

$21 \mathrm{~cm}$ from the axis is at least $50 \%$ lower than when the source is on the axis in all cases. In order

3 to understand these data, the "effective volume" was determined by simulating a grid of ${ }^{216} \mathrm{Po}^{+}$

4 ions with $5 \mathrm{~mm}$ spacing and plotting those that reached either the nozzle or the entrance to petal

5 4. The results are shown in Fig. 6(b), with the ions most likely to be extracted shown as black

6 squares. The effective volume was a narrow cylinder along the central axis, consistent with the

7 fact that moving the source along the axis had a small influence on yield while moving the

8 source slightly off-axis resulted in a significant decrease in yield.

9 The influence of capillary inner diameter was studied using inner diameters of $1 / 16,3 / 32$,

$101 / 8$, and 5/32 inches (corresponding to $1.6,2.4,3.2$, and $4.0 \mathrm{~mm}$, respectively) and a constant

11 length (25 feet, $7.6 \mathrm{~m}$ ). The maximum DP and the corresponding flow rates are presented in

12 Table 3. The capillaries with inner diameters of $1 / 16$ and $3 / 32$ inches had DP not greater than

133.1 torr $(0.41 \mathrm{kPa})$ and flow rates not greater than $1.1 \mathrm{~L} / \mathrm{min}$, so these were not considered

14 further. The efficiencies for the $1 / 8$ inch and 5/32 inch capillaries were qualitatively similar, and

15 both had a flow rate of at least $2.8 \mathrm{~L} / \mathrm{min}$. The $1 / 8$ inch inner diameter capillary was used in

16 most of the following experiments due to its lower maximum flow rate $(2.8 \mathrm{~L} / \mathrm{min})$ but 17 acceptable maximum DP (19 torr, $2.5 \mathrm{kPa})$.

\section{6. Online Characterization}

\section{6.1. Experimental Description}

20 The RTC was characterized in a series of online experiments but the reactions described

21 in Sec. 3 could not be used for this purpose because ${ }^{85} \mathrm{Zr}$ and ${ }^{169} \mathrm{Hf}$ are gamma emitters, and the

22 production cross section of ${ }^{208} \mathrm{~Pb}\left({ }^{50} \mathrm{Ti}, \mathrm{n}\right){ }^{257} \mathrm{Rf}$ is too small to be feasible for characterization 
1 measurements (the largest published cross section is $43_{-8}^{+10} \mathrm{nb}$ [47]). The reaction

$2{ }^{118} \mathrm{Sn}\left({ }^{40} \mathrm{Ar}, 6 \mathrm{n}\right){ }^{152} \mathrm{Er}$ was chosen because a beam of ${ }^{40} \mathrm{Ar}$ was available with sufficient intensity,

$3 \quad{ }^{152}$ Er has a large alpha branch $(\sim 90 \%)$ with a short half-life $(10.3 \pm 0.1 \mathrm{~s})$ [45], and the excitation

4 function has been measured (peak cross section $\sim 100 \mathrm{mb}[48]$ ).

5 A beam of ${ }^{40} \mathrm{Ar}^{5+}$ with an energy of $220 \mathrm{MeV}$ was delivered by the $\mathrm{K} 500$ cyclotron at the

6 Cyclotron Institute at Texas A\&M University. The energy of the beam was measured by passing

7 it through a $48 \mu \mathrm{g} / \mathrm{cm}^{2}{ }^{\text {nat }} \mathrm{C}$ foil, measuring the magnetic rigidity of the resulting charge states,

8 and correcting for energy loss in the foil using SRIM as implemented in LISE++. The beam passed

9 through a $4.5 \mu \mathrm{m} \mathrm{Al}$ degrader before bombarding a $315 \mu \mathrm{g} / \mathrm{cm}^{2}$ enriched ${ }^{118} \mathrm{Sn}$ target. The beam

10 intensity on target was 3-5 pnA. Two collimated Si detectors mounted at $\pm 30^{\circ}$ to the beam axis

11 were used to monitor the intensity of the beam via Coulomb scattering [49], and to ensure that

12 the beam was properly aligned on the target. The recoiling products passed through the same

$13{ }^{\text {nat }} \mathrm{C}$ foil described above in order to equilibrate their charge distribution. These ions entered

14 MARS, which was optimized for transmission of $43.0 \mathrm{MeV}{ }^{152} \mathrm{Er}^{20+}$. A retractable Micron

15 model X-1 16-strip position-sensitive Si detector was mounted at the MARS focal plane. The

16 data acquisition system was substantially similar to the one described in [29]. The incident rate

17 and spatial distribution of ions entering the RTC were optimized using the Si strip detector,

18 which was then retracted. The RTC was mounted $\sim 10 \mathrm{~cm}$ downstream of the strip detector and

19 the VAD with a $5.9 \pm 0.1 \mu \mathrm{m}$ Mylar degrader was mounted just upstream of the strip detector.

20 The estimated error of the VAD angle is $\pm 1^{\circ}$. The RTC entrance window was $2.0 \pm 0.1 \mu \mathrm{m}$

21 Mylar with a $32 \pm 16 \mathrm{~nm} \mathrm{Al} \mathrm{layer} \mathrm{on} \mathrm{each} \mathrm{side,} \mathrm{and} \mathrm{the} \mathrm{MC} \mathrm{pressure} \mathrm{was} 228$ torr (30.4 $\mathrm{kPa})$,

22 although some experiments described below were conducted at vacuum. DP was varied for

23 some experiments but was generally 10 torr $(1.3 \mathrm{kPa})$. The $\mathrm{MC}$ detector was identical to the AC 
1 detector described in Sec. 5, and example energy spectra are shown in Figs. 8(a) and 8(b),

2 respectively. The broad, featureless peak at low-energy observed on the MC detector in Fig. 8(a)

3 is due to the implantation of EVRs and target-like fragments which traversed the separator. This

4 feature is not observed on the AC detector, which has a very clean spectrum, containing only the 5 alpha decays of the primary reaction products.

\section{$6 \quad$ 6.2. Online Extraction Efficiency}

7 The goal of the experiments was to optimize the RTC extraction efficiency as a function

8 of the VAD angle and the electric field distribution. Preliminary measurements with the RTC

9 pressurized but the electric field disabled suggested that the optimum efficiency would be

10 obtained with $\theta \approx 45^{\circ}$. Using this $\mathrm{VAD}$ angle, the electrode potentials were varied

11 systematically while measuring the ratio of the AC detector event rate to a previously measured

12 MC detector rate. Two different measures of absolute extraction efficiency were defined. The

13 first is the fraction of ions $\varepsilon_{\mathrm{RTC}}$ impinging on the RTC window that are ultimately detected by the

$14 \mathrm{AC}$ detector, and the second is the efficiency $\varepsilon_{\mathrm{RTC}}^{\prime}$ that could theoretically be achieved if no ions

15 stopped in the window. Both of these are functions of $\theta$, and are defined by the following

16 equations:

$$
\begin{gathered}
\frac{R_{\mathrm{AC}}(\theta)}{\varepsilon_{\mathrm{AC}} R_{\text {scattering }}(\theta)}=\frac{R_{\mathrm{MC}}\left(0^{\circ}\right)}{\varepsilon_{\mathrm{MC}} R_{\text {scattering }}^{\prime}\left(0^{\circ}\right) f_{\text {range }}\left(0^{\circ}\right) f_{\mathrm{MC}}} \varepsilon_{\mathrm{RTC}}(\theta) \\
\frac{R_{\mathrm{AC}}(\theta)}{\varepsilon_{\mathrm{AC}} R_{\text {scattering }}(\theta)}=\frac{R_{\mathrm{MC}}(\theta)}{\varepsilon_{\mathrm{MC}} R_{\text {scattering }}^{\prime}(\theta) f_{\mathrm{MC}}} \varepsilon_{\mathrm{RTC}}^{\prime}(\theta)
\end{gathered}
$$

19 In both equations, $R_{\mathrm{AC}}$ is the count rate on the $\mathrm{AC}$ detector, $R_{\text {scattering }}$ is the average count rate of

20 the scattering detectors in the target chamber at the time of the $\mathrm{AC}$ detector measurement, $\varepsilon_{\mathrm{AC}}=$

$21(50 \pm 3) \%$ is the intrinsic alpha detection efficiency of the AC detector, $R_{\mathrm{MC}}$ is the count rate on 
1 the MC detector, $\varepsilon_{\mathrm{MC}}=(55 \pm 3) \%$ is the intrinsic alpha detection efficiency of the MC detector,

$2 f_{\text {range }}$ is the fraction of ions which do not range out in the window, and $f_{\mathrm{MC}}=(88 \pm 4) \%$ is the

3 fraction of ions entering the gas whose trajectories would cause them to implant in the MC

4 detector. (See Appendix A for a description of the procedure used to estimate $f_{\mathrm{MC}}$ ). Primes

5 indicate that the scattering rates may be different in temporally separated experiments. The

6 measured $\varepsilon_{\text {RTC }}$ and $\varepsilon_{\text {RTC }}^{\prime}$ data both with and without the electric field on are shown in detail in

7 Fig. 9 as function of the total thickness of Mylar degrader. (The effect of the $32 \pm 16 \mathrm{~nm} \mathrm{Al}$

8 layers on each side of the window was not considered). The maximum values of $\varepsilon_{\mathrm{RTC}}$ and $\varepsilon_{\mathrm{RTC}}^{\prime}$

9 are given in Table 4. When the system is using gas flow only, these are $(21 \pm 3) \%$ and $(25 \pm$

$103) \%$, respectively. When the electric field is on, these are $(35 \pm 4) \%$ and $(44 \pm 5) \%$, respectively.

11 These efficiencies of several tens of percent suggest that the RTC is suitable for a future online

12 chemistry research program. The effects of DP and flow rate on efficiency were also studied.

13 The efficiency was independent of flow rate for $\mathrm{DP}=10$ torr $(1.3 \mathrm{kPa})$. For constant flow rates

14 of $2.5 \mathrm{~L} / \mathrm{min}$, the efficiency increased rapidly with increasing DP to a maximum at DP $=15$ torr

15 (2.0 kPa), followed by a slight decrease above 15 torr, suggesting that a small increase in

16 efficiency could be obtained by increasing DP slightly in future experiments.

17 The measured efficiencies are substantial but also suggest that not all ions are being

18 extracted by the RTC. It is possible that the efficiency is being reduced by a combination of

19 slow transportation times (discussed below), collisions with the electrodes, charge neutralization,

20 and/or molecular ion formation. The optimum biases are given in the fourth column of Table 2

21 (starting with $21.6 \mathrm{~V}$ ), the corresponding electric field is shown in Fig. 10(a), and the calculated

22 effective volume is shown in Fig. 10(b). The latter is centered on the ring region, but this is

23 likely because ions in the window region are being drawn toward the inner chamber groove [see 
1 Fig. 10(a)]. Ions outside the effective volume can potentially collide with other electrodes as

2 well. The effective volume [black squares in Fig. 10(b)] also has an irregular shape compared to

3 the offline simulations [black squares in Fig. 6(b)], suggesting that improvements to the electric

4 field shape could lead to higher efficiency. Additionally, when the ions stopped in the optimum

5 location, the range-out fraction was significant ( 20\%), signifying that the distribution was very

6 broad. Charge neutralization of the ions would eliminate the efficacy of the electric field, but the

7 gas flow has been carefully designed so that neutral atoms can still potentially reach the nozzle, a

8 fact confirmed by the gas flow only data. Studies have indicated that contaminant concentrations

9 as low as $2 \mathrm{ppb}$ are capable of forming molecular ions [41], and the extraction of such ions from

10 a gas stopper has been observed previously (for example, [50, 51]). These molecular ions would

11 be unlikely to have a significant impact on future online chemistry experiments.

12 The transportation time through the system was characterized by fitting the time

13 distribution of ${ }^{152} \mathrm{Er}$ events on the $\mathrm{AC}$ detector at the start of an irradiation to a modified version

14 of Eq. (1) in [52]. If $\tau_{\mathrm{RTC}}$ is the extraction time of the system, then for time $t>\tau_{\mathrm{RTC}}$,

$$
N(t)=\frac{\varepsilon \sigma N_{\text {target }} I}{\lambda+\tau_{\mathrm{RTC}}^{-1}}\left\{1-\exp \left[-\left(\lambda+\tau_{\mathrm{RTC}}^{-1}\right)\left(t-\tau_{\mathrm{RTC}}\right)\right]\right\},
$$

16 where $N$ is the number of nuclei at time $t, \varepsilon$ is the efficiency for transmitting ions to the AC

17 detector (including all facets of separator efficiency and RTC efficiency), $\sigma$ is the nuclear

18 reaction cross section, $N_{\text {target }}$ is the areal density of nuclei in the target, $I$ is the intensity of the

19 beam, and $\lambda$ is the nuclide's decay constant. The measured extraction times are given in Table 2

20 for a variety of experimental conditions. When tuned for optimum efficiency, the transportation

21 times were determined to be $8.5 \pm 1.0 \mathrm{~s}$ (gas flow only) and $6.9 \pm 0.6 \mathrm{~s}$ (gas flow and electric

22 field). These are sufficient for online chemistry experiments, but an even higher extraction 
1 efficiency could be achieved if the nuclide under study had a half-life much greater than $\tau_{\mathrm{RTC}}$.

2 (Online chemistry experiments often utilize nuclides with half-lives greater than $1 \mathrm{~min}$, but the

3 half-life of ${ }^{152} \mathrm{Er}$ is only $10.3 \mathrm{~s}$ ). This theoretical extraction efficiency $\varepsilon_{\mathrm{RTC}}^{\prime \prime}$ is defined as

$$
\varepsilon_{\mathrm{RTC}}^{\prime \prime}(\theta)=\varepsilon_{\mathrm{RTC}}^{\prime}(\theta) \cdot \exp \left(+\lambda \tau_{\mathrm{RTC}}\right),
$$

5 and optimum values are given in Table 4. These data suggest that a very high efficiency [(70 \pm 6 9)\%] may be possible.

7 The design of the current system falls between relative simplicity of "gas flow only"

8 RTCs in use at laboratories worldwide [10-12] and the more complex designs that use a variety

9 of oscillating electric fields [5, 9, 13-18]. Efficiencies for the former are not widely reported, but

10 Ref. [12] reports that the extraction efficiency for one device is $(80 \pm 8) \%$. The nominal

11 performance of the current device was within a factor of 2 of this efficiency, and further

12 improvements (discussed below) could reduce this disparity. Although it was not tested in the

13 current work, the use of electric fields to focus ions into a small extraction nozzle would likely

14 make the current system suitable for injecting ions into a secondary analysis system, such as the

15 FIONA mass spectrometer. The RF systems described above are used routinely for similar 16 purposes, but some require thousands of individual pieces. The current system has substantially

17 fewer components, making it easier to fabricate and maintain. Regardless, the use of electric

18 fields adds substantially to the development time and creates a large number of "knobs" that

19 must be optimized across a large parameter space. The benefits of the use of electric fields must

20 be considered carefully when an RTC will be used to provide activity for chemistry experiments, 21 since a highly optimized "gas flow only" RTC can have a high efficiency. 


\section{7. Future Work}

The data suggest that the efficiency is being reduced by collisions with the electrodes,

3 range-out of ions in the window, and slow transportation times. The incident energy distribution

4 of the ions was broad, resulting in some ions colliding with the flower petal electrodes before

5 thermalization and others ranging out in the window. These facts suggest that increased $\varepsilon_{\text {RTC }}^{\prime}$

6 could be obtained in future experiments by increasing the gas pressure to narrow the stopped

7 distribution of ions. Fig. 10(a) also shows that the electric field in the window region was

8 guiding ions toward the inner chamber groove, so a biased wire mesh covering for the groove is

9 currently being designed to minimize this effect. The mesh was simulated in SIMION using the

10 biases given in the final column of Table 2, and the corresponding electric field and effective

11 volume are shown in Fig. 11. The effective volume is substantially increased in size and

12 uniformity, and is closer to the intended performance of the system. The field strengths are also

13 higher, which should result in faster extraction of ions.

\section{8. Conclusions}

A new RTC has been successfully designed, fabricated, and commissioned at Texas

16 A\&M University. The design is based on the gas stopper that was in routine use at NSCL with

17 modifications for the much smaller ranges of the ions of interest in the current work. These ions

18 first pass through a variable angle degrader that controls their thermalized distribution in a He-

19 filled chamber with a pressure of 228 torr $(30.4 \mathrm{kPa})$. A series of electrodes creates a carefully

20 shaped electric field that draws the ions toward another set of electrodes that focuses them into

21 an extraction nozzle. A maximum extraction efficiency of (35 \pm 4$) \%$ was measured directly, and

22 the performance of the RTC is sufficient for a planned online chemistry research program. 


\section{Acknowledgements}

The authors would like to thank S. L. Molitor and W. T. Seward for their skillful design

3 and fabrication of the RTC, respectively; D. J. Morrissey for providing the flower petal and

4 nozzle assembly that was incorporated into the design; the Cyclotron Institute accelerator group

5 and operations staff for delivering the beams of ${ }^{40} \mathrm{Ar}$; and the reviewer for informative

6 comments. This work was supported by the Welch Foundation under grant number A-1710.

7 This material is based upon work supported by the U.S. Department of Energy, Office of

8 Science, Office of Nuclear Physics under Award Numbers DE-FG02-93ER40773, DE-FG02-

9 12ER41869/DE-SC0008126, and DE-FG07-05ID14692/MUSC09-100. Finally, this work was

10 supported by the U.S. National Science Foundation under award number PHY-1004780.

\section{Appendix A. Measurement of $f_{\mathrm{MC}}$}

12 The measurement of $f_{\mathrm{MC}}$ required that $f_{\text {screen }}$ and $f_{\text {grid }}$ be measured first, so the count rate

13 measured on the MC detector was modeled as the product of several quantities:

$$
\frac{R_{\mathrm{MC}}(\theta)}{\varepsilon_{\mathrm{MC}} R_{\text {scattering }}(\theta)}=\frac{R_{\text {strip }}\left(0^{\circ}\right)}{\varepsilon_{\text {geom }} \varepsilon_{\text {strip }} R_{\text {scattering }}^{\prime}\left(0^{\circ}\right)} f_{\text {screen }} f_{\text {grid }} f_{\text {holder }} f_{\text {range }}(\theta) f_{\mathrm{MC}},
$$

15 where $R_{\text {strip }}$ is the count rate on the strip detector, $\varepsilon_{\text {geom }}$ is its geometrical efficiency, $\varepsilon_{\text {strip }}$ is its 16 alpha detection efficiency, $f_{\text {screen }}$ is the fraction of ions which pass though the protector screen,

$17 f_{\text {grid }}$ is the fraction of ions which pass through the support grid, and $f_{\text {holder }}$ is the fraction of ions

18 which are NOT blocked geometrically by the window holder. Other quantities are as defined in

19 Sec. 6.2. The prime indicates that the scattering rates may not be equal in temporally separated

20 experiments. The position distribution of ions implanting in the strip detector suggests that $\varepsilon_{\text {geom }}$

$21 \approx 100 \%$, and its alpha detection efficiency was estimated to be $\varepsilon_{\text {strip }}=(55 \pm 3) \%$. 
In order to measure $f_{\text {screen }}$ and $f_{\text {grid }}$, the size of the product beam was reduced by closing

2 the final MARS aperture ("slits") to $\pm 0.5 \mathrm{~cm}$ in both the horizontal and vertical directions,

3 setting $\theta=0^{\circ}$, and removing the gas from the RTC. Under these conditions, it was assumed that

$4 \quad 100 \%$ of ions exiting the spectrometer would pass through the window holder and that all ions

5 passing through the window would implant in the MC detector, i.e., $f_{\text {holder }}=f_{\text {range }}\left(0^{\circ}\right)=f_{\mathrm{MC}}=1$.

6 The strip detector count rate was considered to be the true rate of ions incident on the front of the

7 RTC, so the ratio of MC detector rate to strip detector rate gives the product $f_{\text {screen }} f_{\text {grid }}=(71 \pm$

86 ) $\%$. (Based on geometry alone, one would estimate $f_{\text {screen }} \approx 88 \%$ and $f_{\text {grid }} \approx 81 \%$, which gives

$9 f_{\text {screen }} f_{\text {grid }} \approx 71 \%$ ).

10 These results allowed $f_{\text {holder }}$ and $f_{\mathrm{MC}}$ to be measured. The slits were fully re-opened with

$11 \theta=0^{\circ}$. Again assuming that $f_{\text {range }}\left(0^{\circ}\right)=1$, the product $f_{\text {holder }} f_{\text {MC }} \approx 87 \%$ could be determined

12 because all of the other quantities in Eq. (6) were measured at $\theta=0^{\circ}$. The experiments with the

13 slits closed showed that the beam expands spatially as it traversed the distance from the slits to

14 the strip detector. $f_{\text {holder }}$ and $f_{\mathrm{MC}}$ are geometrical factors that depend only on the ions' trajectories

15 (since there are no magnets downstream of the slits), so they can be simulated based on the

16 measured distribution of ions on the strip detector and an assumed spatial magnification of the

17 beam. The magnification was varied until the product $f_{\text {holder }} f_{\text {MC }}$ agreed with the experimentally

18 determined value. The fixed magnification and the known value of $f_{\text {holder }} f_{\text {MC }}$ provided two

19 constraints that allowed $f_{\text {holder }}$ and $f_{\mathrm{MC}}$ to be estimated individually. The results of this procedure

20 were $f_{\text {holder }}=(99 \pm 4) \%$ and $f_{\mathrm{MC}}=(88 \pm 4) \%$.

\section{10. References}

22 [1] R. Eichler, et al., Radiochim. Acta 98 (2010) 133.

23 [2] R. Eichler, et al., Nature (London) 447 (2007) 72. 
1 [3] Ch. E. Düllmann, et al., Nature (London) 418 (2002) 859.

[4] R. Eichler, et al., Nature (London) 407 (2000) 63.

[5] J.B. Neumayr, et al., Nucl. Instrum. Meth. B 244 (2006) 489.

[6] M. Block, et al., Nucl. Instrum. Meth. B 266 (2008) 4521.

[7] A. Chaudhuri, et al., Appl. Phys. B 114 (2014) 99.

[8] G. Savard, et al., Nucl. Instrum. Meth. B 266 (2008) 4086.

[9] S. Schwarz, et al., Nucl. Instrum. Meth. B 317 (2013) 463.

[10] H. Haba, et al., J. Nucl. Radiochem. Sci. 8 (2007) 55.

[11] U.W. Kirbach, et al., Nucl. Instrum. Meth. A 484 (2002) 587.

[12] J. Even, et al., Nucl. Instrum. Meth. A 638 (2011) 157.

[13] G. Savard, J. Phys. Conf. Ser. 312 (2011) 052004.

[14] G. Savard, et al., Nucl. Instrum. Meth. B 204 (2003) 582.

[15] C. Droese, et al., Nucl. Instrum. Meth. B 338 (2014) 126.

[16] M. Ranjan, et al., Europhys. Lett. 96 (2011) 52001.

[17] M. Brodeur, et al., Int. J. Mass Spectrom. 336 (2013) 53.

[18] F. Arai, et al., Int. J. Mass Spectrom. 362 (2014) 56.

[19] T. Sonoda, et al., AIP Conf. Proc. 1104 (2009) 132.

[20] L. Weissman, et al., Nucl. Phys. A 746 (2004) 655c.

[21] L. Weissman, et al., Nucl. Instrum. Meth. A 522 (2004) 212.

[22] L. Weissman, et al., Nucl. Instrum. Meth. A 531 (2004) 416.

[23] L. Weissman, et al., Nucl. Instrum. Meth. A 540 (2005) 245.

[24] P.A. Lofy, Ph.D. Thesis, Michigan State University (2003).

[25] M. Block, et al., Eur. Phys. J. D 45 (2007) 39.

[26] Ch. E. Düllmann, et al., Nucl. Instrum. Meth. A 551 (2005) 528.

[27] R.E. Tribble, R.H. Burch, and C.A. Gagliardi, Nucl. Instrum. Meth. A 285 (1989) 441.

[28] R.E. Tribble, C.A. Gagliardi, and W. Liu, Nucl. Instrum. Meth. B 56/57 (1991) 956.

[29] C.M. Folden III, et al., Nucl. Instrum. Meth. A 678 (2012) 1.

[30] R. Sudowe, et al., Radiochim. Acta 94 (2006) 123.

[31] Ch. E. Düllmann, et al., Radiochim. Acta 97 (2009) 403.

[32] J.P. Omtvedt, et al., J. Nucl. Radiochem. Sci. 3 (2002) 121.

[33] L. Stavsetra, et al., Nucl. Instrum. Meth. A 543 (2005) 509.

[34] O.B. Tarasov and D. Bazin, Nucl. Phys. A 746 (2004) 411.

[35] J.F. Ziegler, M.D. Ziegler, and J.P. Biersack, Nucl. Instrum. Meth. B 268 (2010) 1818.

[36] J.F. Ziegler, Computer Code SRIM-2010, available at http://www.srim.org.

[37] Computer Code SIMION, available at http://www.simion.com.

[38] A.D. Appelhans and D.A. Dahl, Int. J. Mass Spectrom. 244 (2005) 1.

[39] R. Johnsen and M.A. Biondi, J. Chem. Phys. 57 (1972) 5292.

[40] CD-adapco, Computer Code STAR-CCM+, available at http://www.cdadapco.com/products/star-ccm.

[41] M. Facina, et al., Nucl. Instrum. Meth. B 266 (2008) 4471.

[42] P. Schury, et al., Phys. Rev. C 75 (2007) 055801.

[43] National Instruments, Computer Code LABVIEw, available at http://www.ni.com/labview.

[44] A.J. Howard, Nucl. Instrum. Meth. B 73 (1993) 53.

[45] National Nuclear Data Center (2015), available at http://www.nndc.bnl.gov.

[46] K. Hámori, M. Váradi, and J. Csikai, Appl. Radiat. Isotopes 64 (2006) 854.

46 [47] I. Dragojević, et al., Phys. Rev. C 78 (2008) 024605. 
[48] H. Gauvin, et al., Phys. Rev. C 10 (1974) 722.

[49] E. Segrè, in Nuclei and Particles: An Introduction to Nuclear and Subnuclear Physics (W.

3 A. Benjamin, Inc., Reading, Massachusetts, 1977), pp. 26-27.

4 [50] M. Block, et al., Phys. Rev. Lett. 100 (2008) 132501.

5 [51] R. Ringle, et al., Phys. Rev. C 75 (2007) 055503.

6 [52] R. Dressler, et al., Transport Time Optimization of IVO: Part II: Model Calculations, in PSI Annual Report 2006 (2007), p. 7, available at http://www.psi.ch/lch/AnnualReportsEN/PSI_LCH_AnnualReport2006.pdf, accessed 2-32015. 
Figures

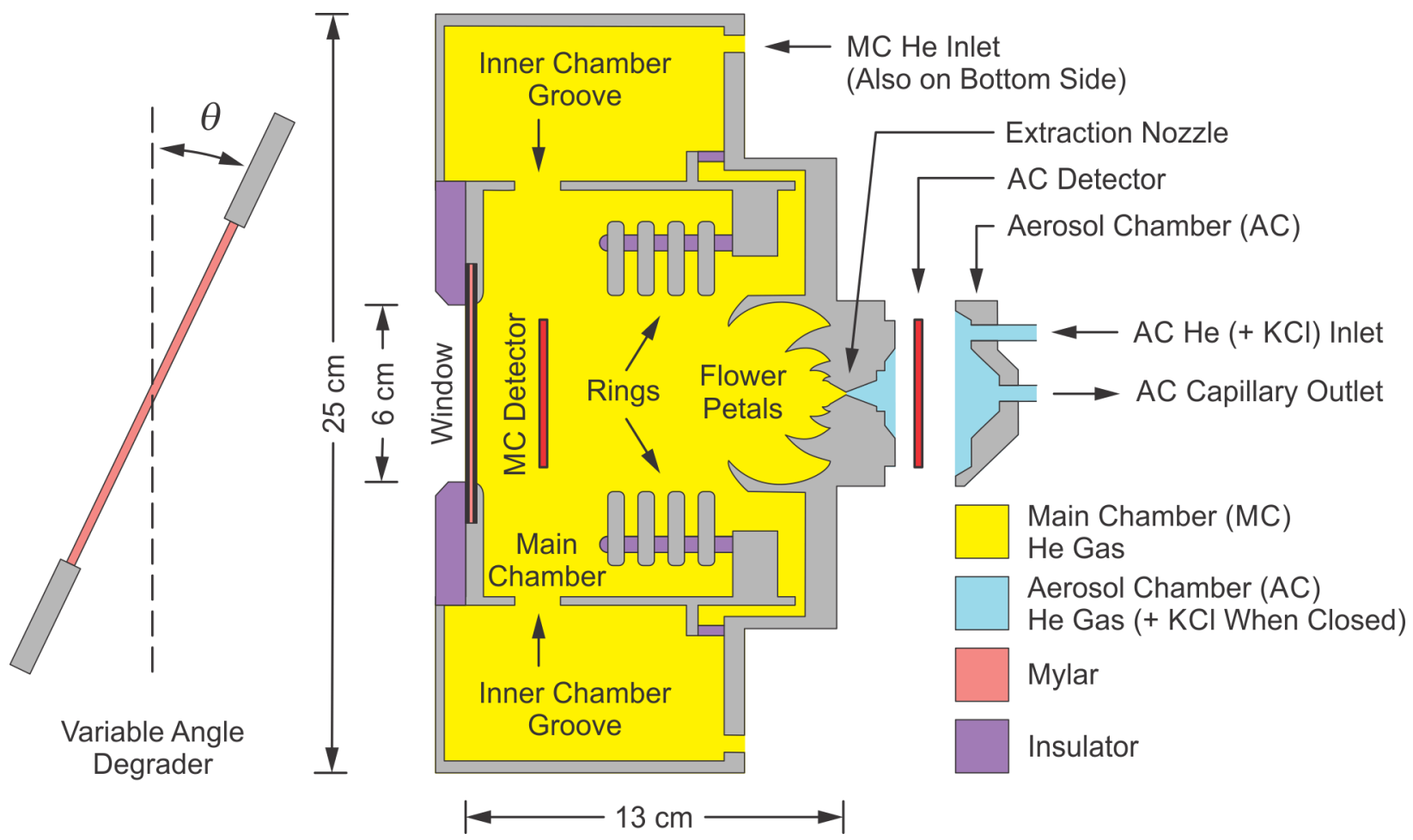

3 Fig. 1. Schematic of the RTC. Ions enter from the left and pass through the variable angle

4 degrader before entering the window. Yellow color indicates pure $\mathrm{He}$ in the main chamber 5 (MC), while blue indicates $\mathrm{He}$ gas possibly laden with $\mathrm{KCl}$ aerosols in the aerosol chamber (AC) 6 when it is closed. Both detectors are shown inserted, which requires that the AC be retracted. 7 Black lines on the window indicate the Al layer. Some non-essential pieces have been removed 8 for clarity. 


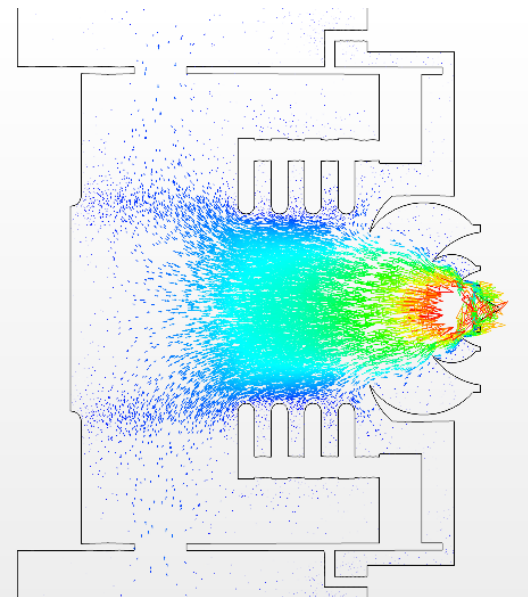

(a)

Velocity $(\mathrm{m} / \mathrm{s})$

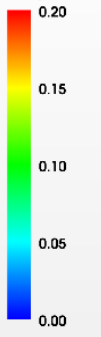

1

Fig. 2. (a) STAR-CCM+ simulation of the gas flow in the RTC. (b) Detail of the gas flow in the 3 nozzle region. Note that the scales are different between the two panels. 

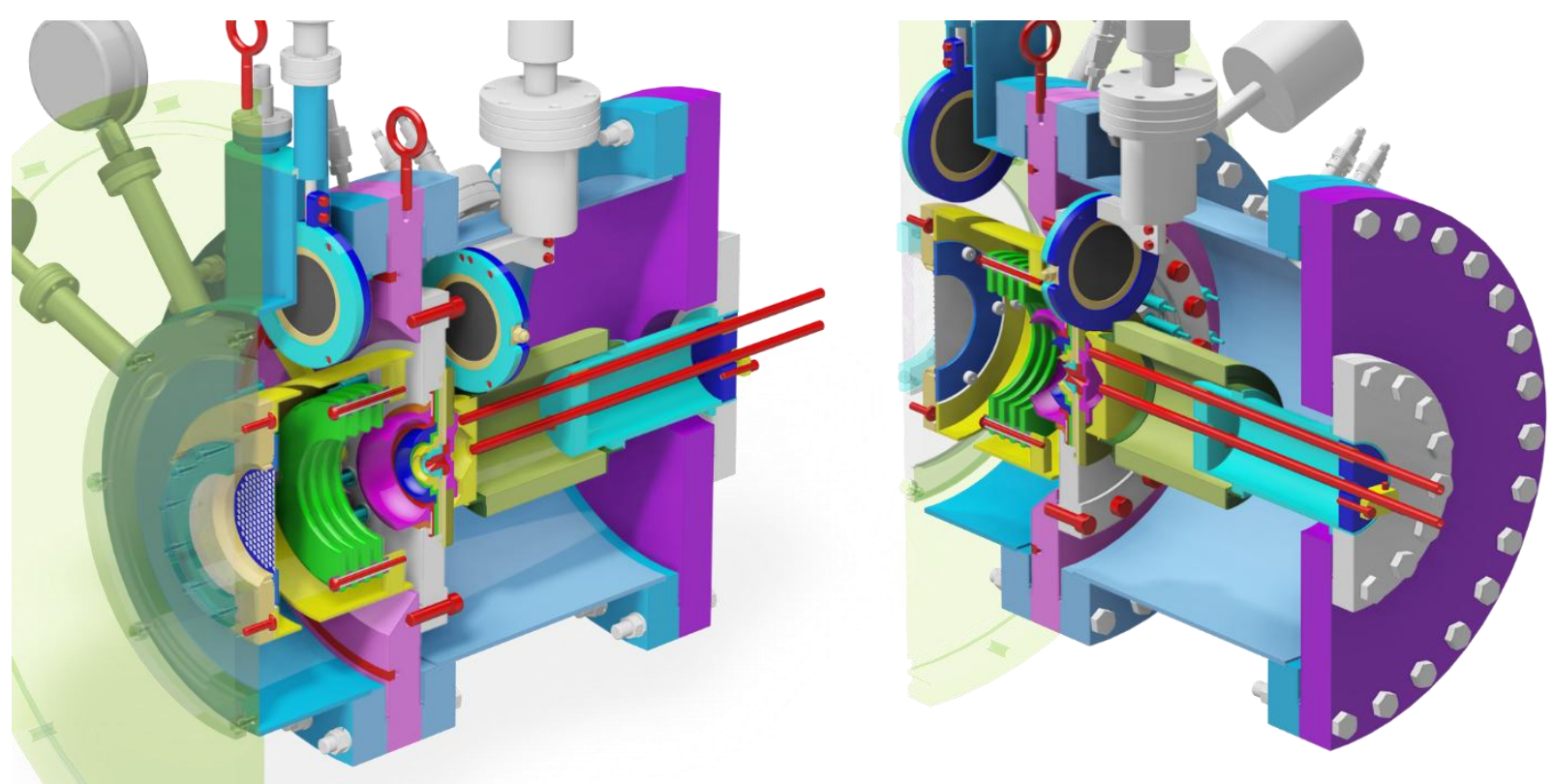

Fig. 3. Isometric views of the RTC. The flange shown in transparent yellow mounts to the detector chamber of the MARS spectrometer. The ring electrodes are shown in green and the "flower petal" electrodes have multiple colors with the first petal shown in purple. Ruggedized Si detectors (brown circles in blue holders) are shown in their retracted positions for clarity of presentation. The AC chamber and bellows are shown in their forward position, although they were retracted for the experiments in the current work. Non-essential elements have been omitted from the figures in order to show detail, including the VAD. Left: Front view of the RTC. Ions enter from the left through the protector screen, the grid, and the window. Right: Rear view of the RTC. Gas enters and exits the AC chamber through the tubes shown in red. 


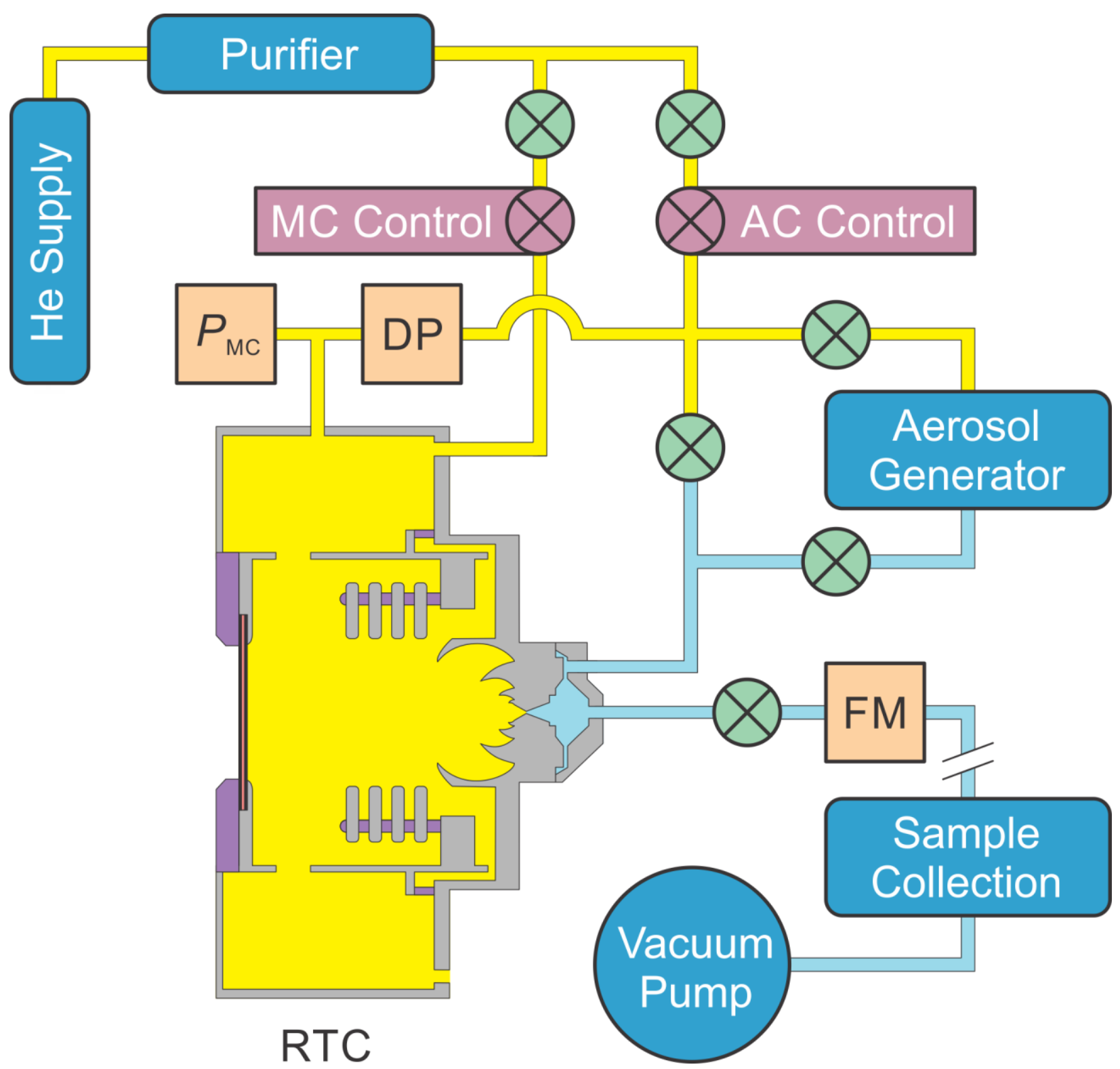

Fig. 4. Gas-handling system of the RTC. Color-coding of the gas is the same as in Fig. 1. Circles with an " $\mathrm{X}$ " are valves, and the $\mathrm{MC}$ and $\mathrm{AC}$ automated pressure control valves are as indicated. " $P_{\mathrm{MC}}$ " is a transducer that measures the absolute pressure in the MC, "DP" is a differential pressure transducer that measures the difference in pressure between the MC and $\mathrm{AC}$, and "FM" is a flow meter that measures the total flow of He out of the system. In the current work, the aerosol generator was bypassed and there was no sample collection. 


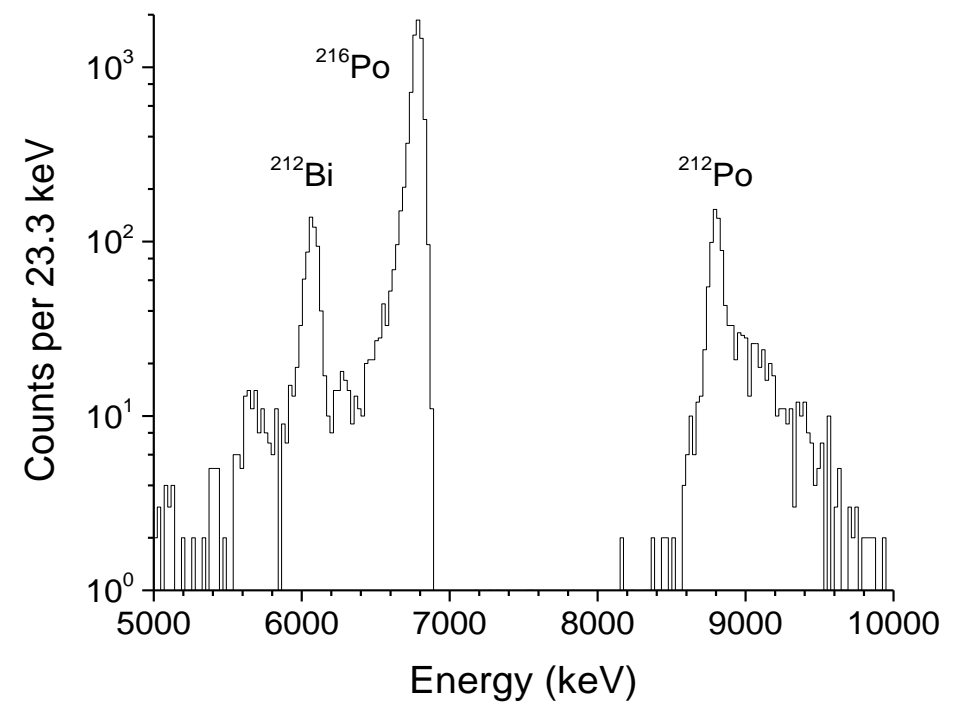

2 Fig. 5. Example alpha spectrum observed on the AC detector from a ${ }^{228} \mathrm{Th}$ source mounted in 3 the MC. Note that none of the parent nuclides of ${ }^{216} \mathrm{Po}$ are observed in the spectrum. 

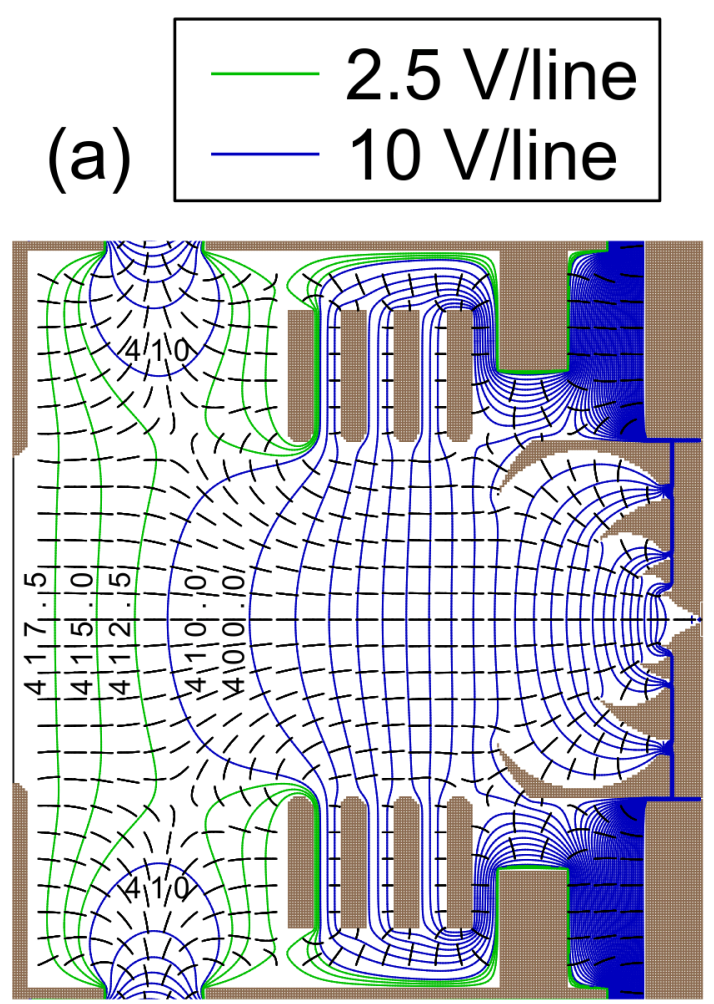

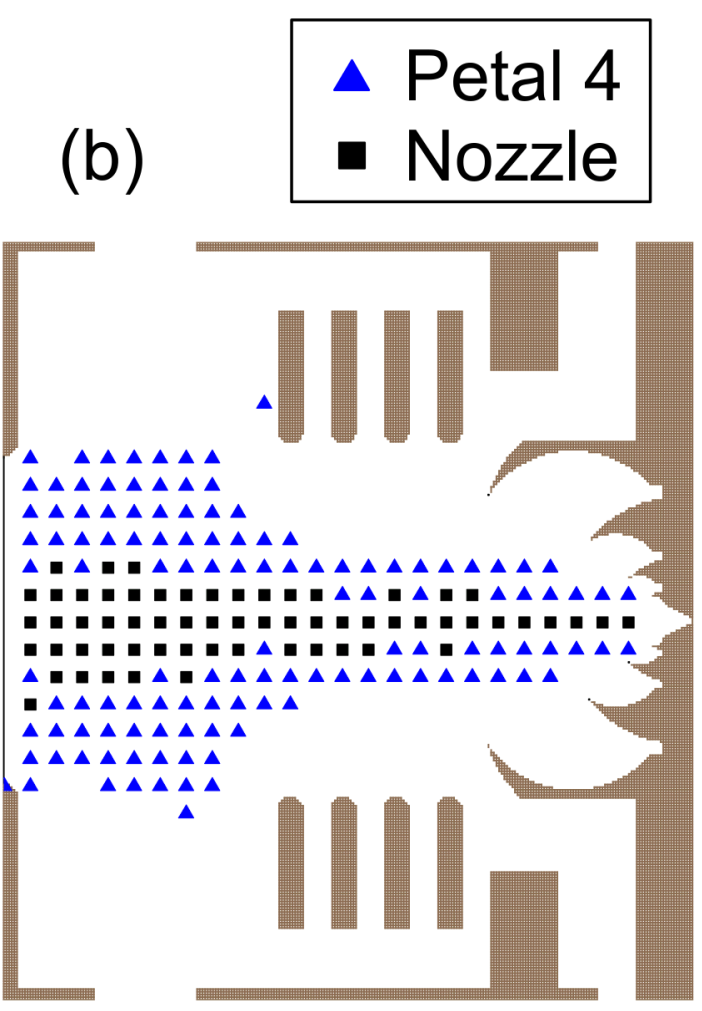

Fig. 6. SIMION simulations of the conditions that gave the optimum extraction of ${ }^{216}$ Po in offline experiments. Electrodes are shown in brown and insulators are not shown. (a) The electric field. The biases applied to the electrodes are given in the seventh column of Table 2 (starting with 420 V). Equipotential lines are shown as colored solid lines and some are labeled in volts for clarity. The difference between each equipotential line is $10 \mathrm{~V}$ for blue lines and $2.5 \mathrm{~V}$ for green lines. Black dashes show the motion of particles due to the electric field only and approximate the electric field lines. (b) Effective volume under the conditions in panel (a). A grid of ${ }^{216} \mathrm{Po}^{+}$ions with $5 \mathrm{~mm}$ spacing in each direction was simulated. Black squares indicate ions that reached the entrance to the nozzle, and blue triangles indicate ions that reached the entrance to flower petal 4 , which is $2 \mathrm{~mm}$ upstream of the nozzle. See the main text for a discussion. 


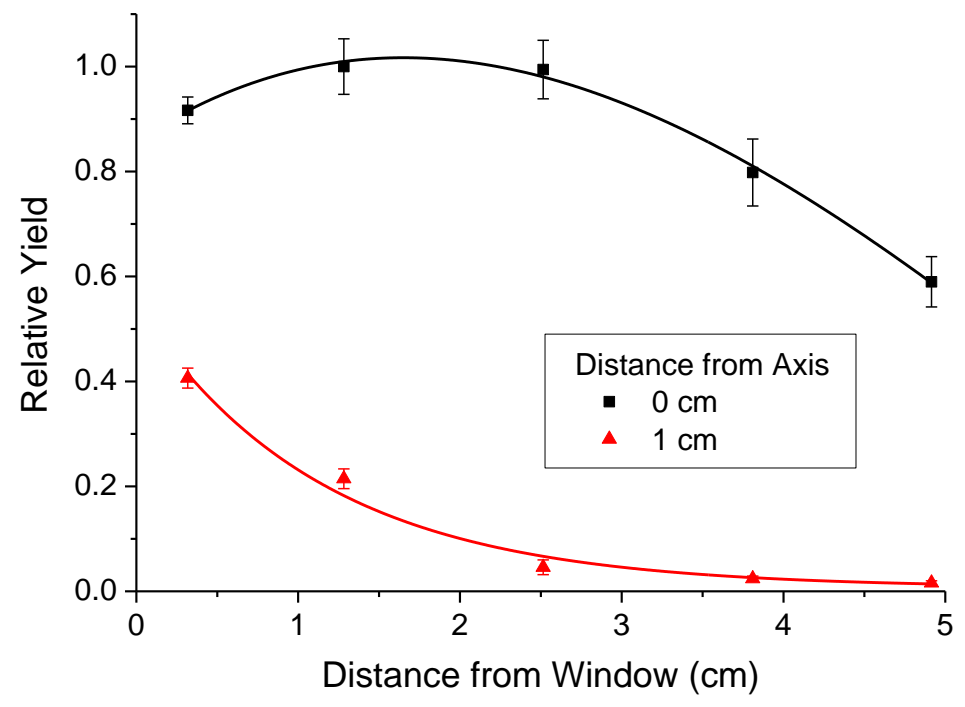

1

Fig. 7. Extraction yield of ${ }^{216}$ Po produced from a ${ }^{228}$ Th source as a function of position within the RTC. The source was located either on the axis (black squares) or $1 \mathrm{~cm}$ from the central axis (red triangles). These data are not corrected for decay losses because the transportation time could not be measured directly. Lines are intended to guide the eye. 

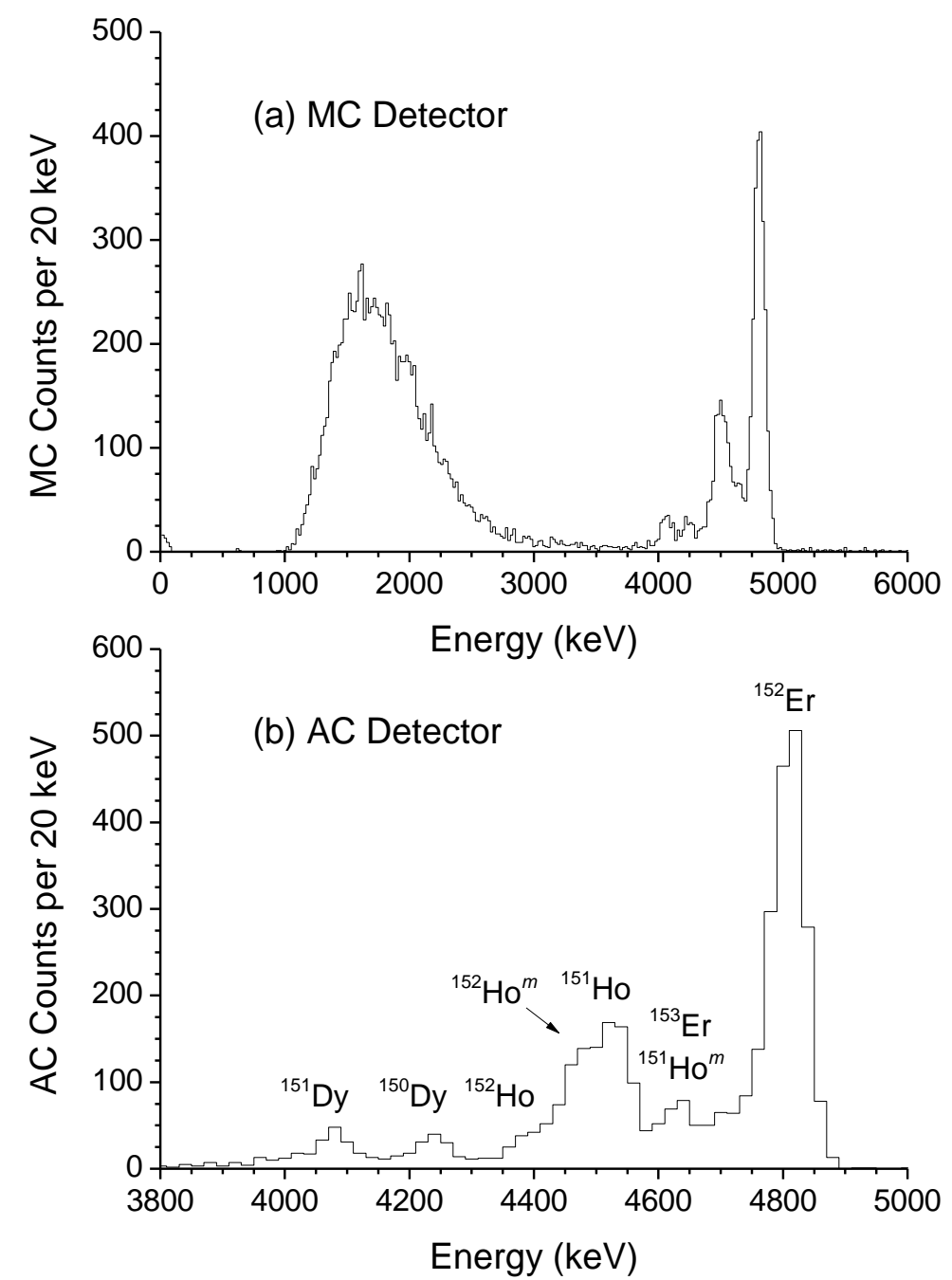

3 Fig. 8. (a) Energy spectrum observed on the MC Si detector with a total Mylar degrader 4 thickness of $10.3 \pm 0.2 \mu \mathrm{m}\left(\theta=45^{\circ}\right)$. The broad, featureless peak from $1000-3000 \mathrm{keV}$ is due to 5 implanting EVRs and target-like fragments which traversed the spectrometer. (b) Example 6 alpha spectrum observed on the AC Si detector. The spectrum from 4000-5000 keV in panel (a) 7 is substantially similar to the same range in panel (b), and the nuclide assignments apply equally 8 to both panels. 


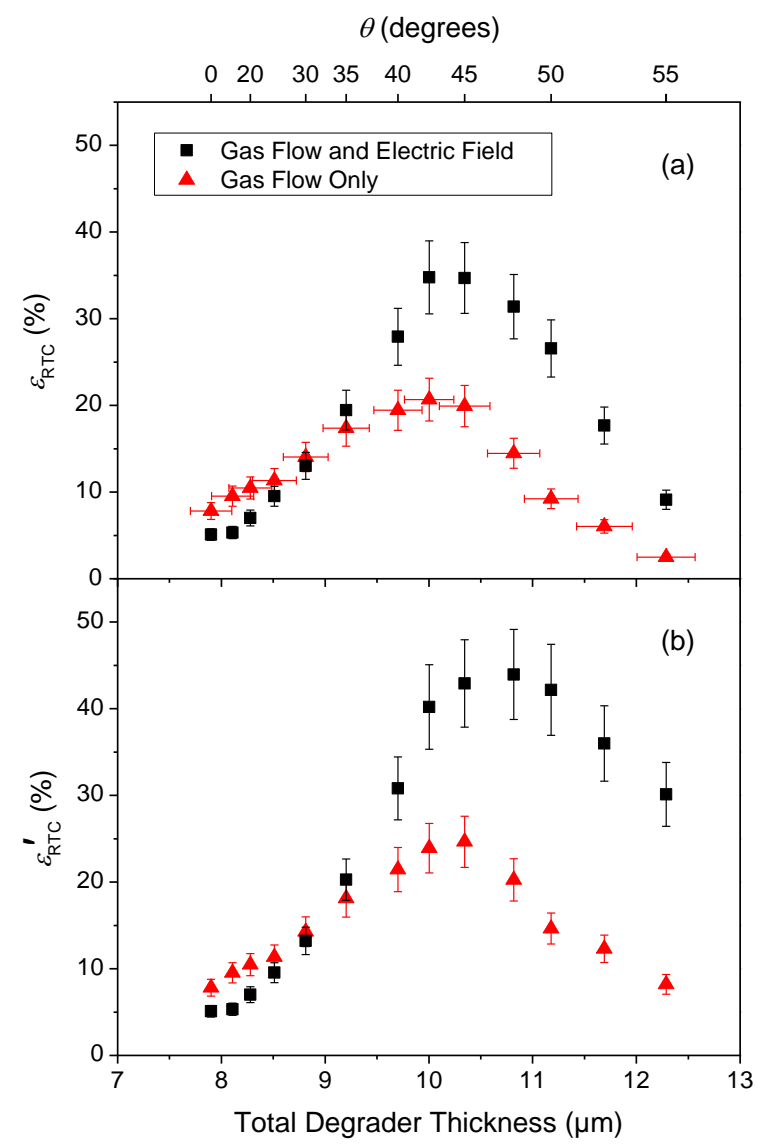

Fig. 9. Measured extraction efficiencies of the RTC as a function of the total thickness of the two Mylar degraders. Data were collected under conditions where the electric fields were both on (black squares) and off (red triangles). These data are not corrected for decay losses during transportation. In all cases, the MC pressure was 228 torr $(30.4 \mathrm{kPa})$, the flow rate was 2.5 $\mathrm{L} / \mathrm{min}$, and DP was 10 torr $(1.3 \mathrm{kPa})$. Horizontal error bars are only shown on one data set for clarity but are comparable for all data sets. (a) Efficiency measured as the ratio of ions detected after the nozzle to the number incident on the front window. See Eq. (2) for the definition of $\varepsilon_{\mathrm{RTC}}$. (b) The same as the upper panel, except corrected for the fraction of ions that do not enter 10 the gas. See Eq. (3) for the definition of $\varepsilon_{\mathrm{RTC}}^{\prime}$. 

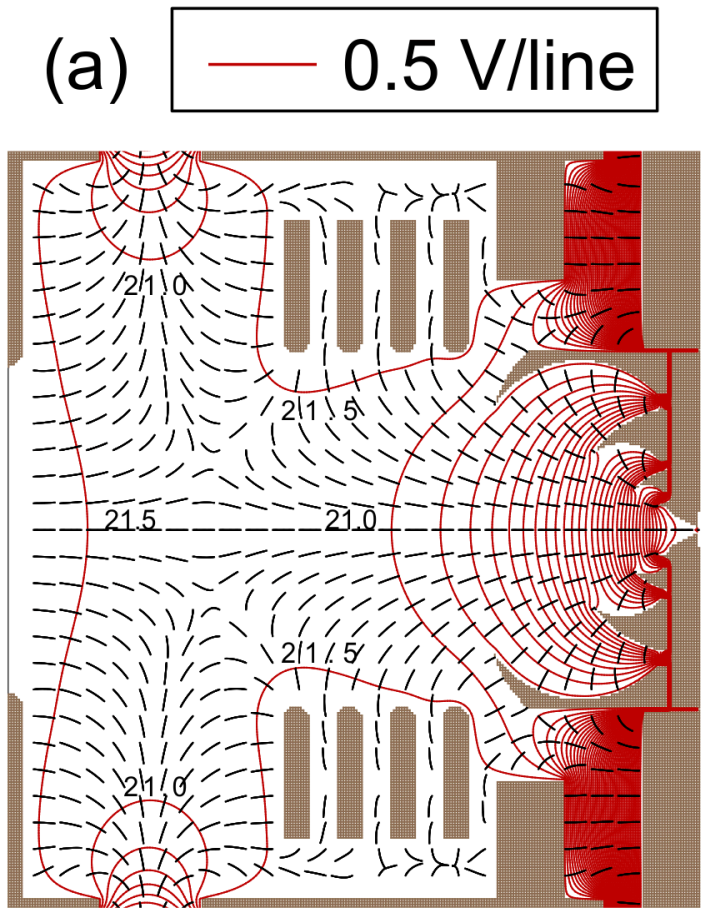

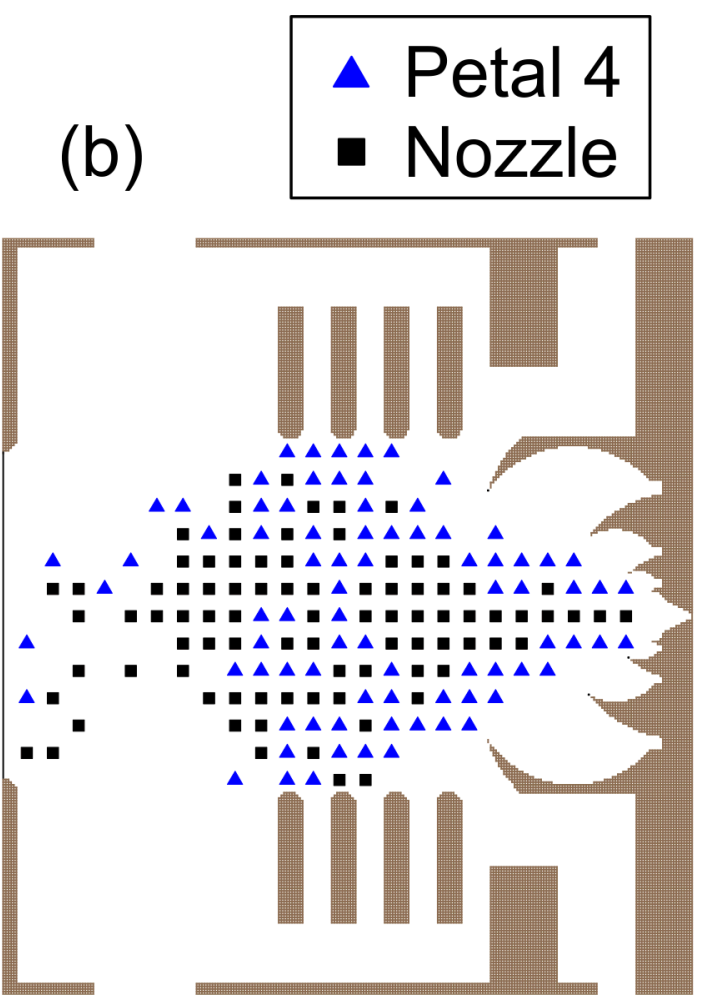

Fig. 10. SIMION simulations of the conditions that gave the optimum extraction of ${ }^{152} \mathrm{Er}$ in online experiments. Electrodes are shown in brown and insulators are not shown. (a) The electric field. The biases applied to the electrodes are given in the fourth column of Table 2 (starting with 21.6 V). Equipotential lines are shown as solid red lines and some are labeled in volts for clarity. The difference between each equipotential line is $0.5 \mathrm{~V}$. Black dashes show the motion of particles due to the electric field only and approximate the electric field lines. (b) Effective volume under the conditions in panel (a). A grid of ${ }^{152} \mathrm{Er}^{+}$ions with $5 \mathrm{~mm}$ spacing in each direction was simulated. Black squares indicate ions that reached the entrance to the nozzle, and blue triangles indicate ions that reached the entrance to flower petal 4, which is $2 \mathrm{~mm}$ upstream of the nozzle. See the main text for a discussion. 

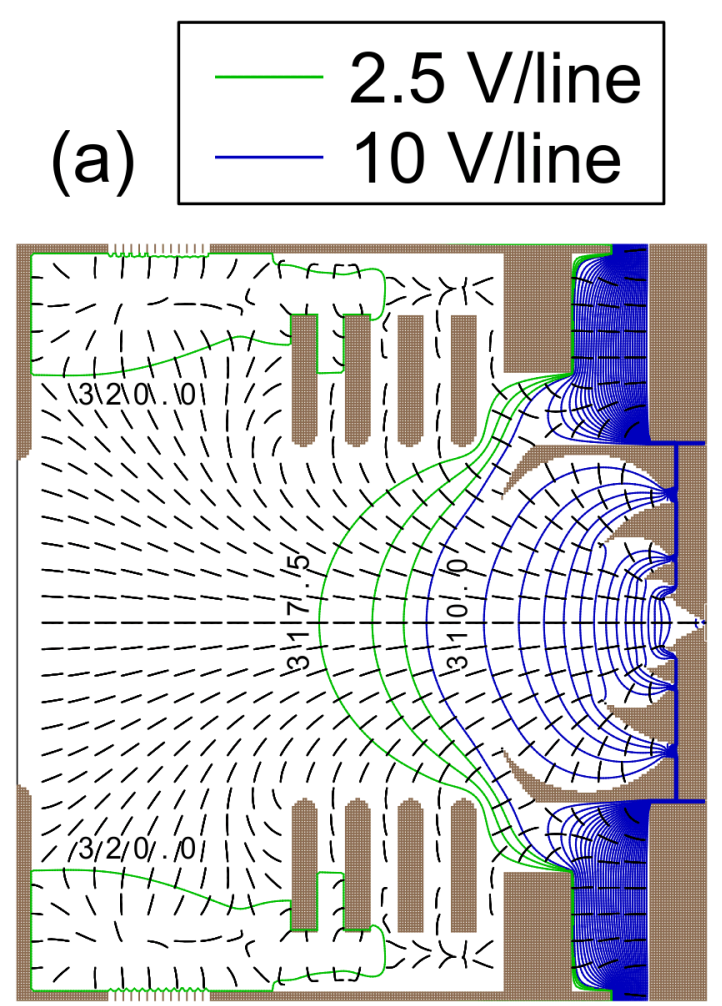
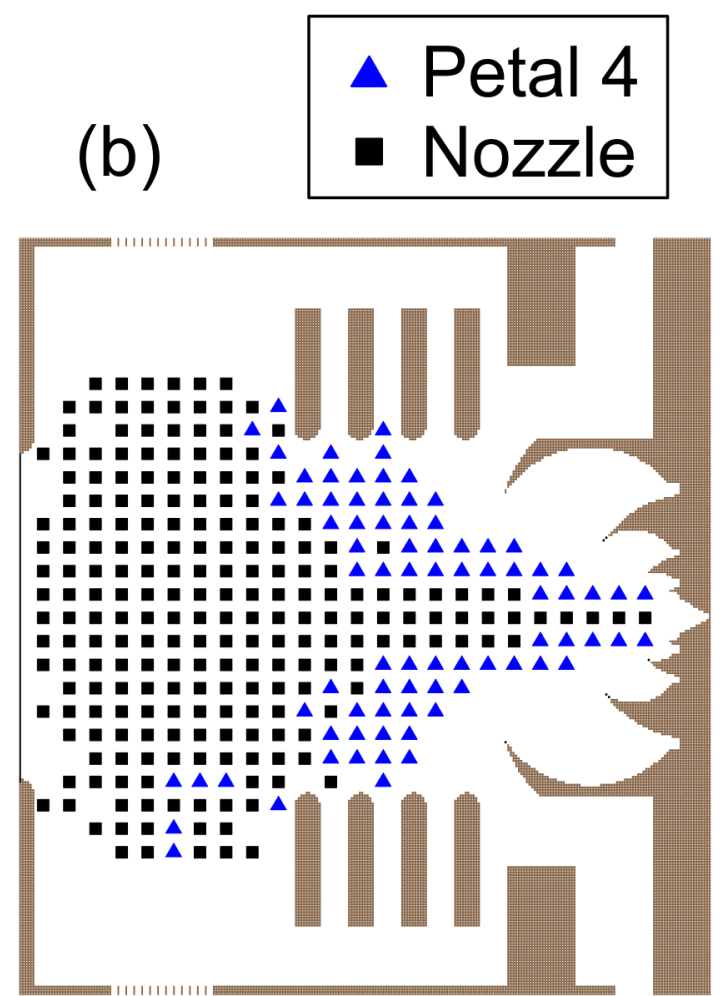

Fig. 11. SIMION simulations of the RTC with a proposed mesh grid around the inner chamber groove. Electrodes are shown in brown and insulators are not shown. (a) The electric field. The biases applied to the electrodes are given in the final column of Table 2 (starting with $320 \mathrm{~V}$ ). Equipotential lines are shown as colored solid lines and some are labeled in volts for clarity. The difference between each equipotential line is $10 \mathrm{~V}$ for blue lines and $2.5 \mathrm{~V}$ for green lines. Black dashes show the motion of particles due to the electric field only and approximate the electric field lines. (b) Effective volume under the conditions in panel (a). A grid of ${ }^{152} \mathrm{Er}^{+}$ions with 5 $\mathrm{mm}$ spacing in each direction was simulated. Black squares indicate ions that reached the entrance to the nozzle, and blue triangles indicate ions that reached the entrance to flower petal 4, which is $2 \mathrm{~mm}$ upstream of the nozzle. See the main text for a discussion. 
Tables

2 Table 1. Properties of reactions used to simulate the RTC. Targets were assumed to have a 3 thickness of $500 \mu \mathrm{g} / \mathrm{cm}^{2}$. EVR energies are given after a $\sim 50 \mu \mathrm{g} / \mathrm{cm}^{2}{ }^{\text {nat }} \mathrm{C}$ stripper foil located 4 immediately downstream of the target. Column 4 gives the required EVR energy to stop the ions $5 \quad 2.5 \mathrm{~cm}$ deep in the $\mathrm{He}$ gas when the pressure is 228 torr $(30.4 \mathrm{kPa})$. Spatial distributions are 6 incident on the RTC window. Reduced mobilities were estimated using Ref. [39].

\begin{tabular}{ccccccc}
\hline & & $\begin{array}{c}\text { EVR Energy } \\
\text { After Stripper } \\
(\mathrm{MeV})\end{array}$ & $\begin{array}{c}\text { EVR Energy } \\
\text { After Window } \\
(\mathrm{MeV})\end{array}$ & $\begin{array}{c}\text { Horizontal } \\
\text { FWHM } \\
(\mathrm{mm})\end{array}$ & $\begin{array}{c}\text { Vertical } \\
\text { FWHM } \\
(\mathrm{mm})\end{array}$ & $\begin{array}{c}\text { Estimated } \\
\text { Reduced } \\
\text { Rebility } \\
\left(\mathrm{cm}^{2} \mathrm{~V}^{-1} \mathrm{~s}^{-1}\right)\end{array}$ \\
\hline${ }^{70} \mathrm{Ge}\left({ }^{18} \mathrm{O}, 3 \mathrm{n}\right)$ & ${ }^{85} \mathrm{Zr}$ & $12.5 \pm 1.9$ & $1.4 \pm 0.3$ & $-{ }^{\mathrm{a}}$ & 17.2 & 15.9 \\
${ }^{124} \mathrm{Sn}\left({ }^{50} \mathrm{Ti}, 5 \mathrm{n}\right)$ & ${ }^{169} \mathrm{Hf}$ & $53.6 \pm 3.1$ & $2.6 \pm 1.1$ & $-{ }^{20}$ & 13.1 & 15.7 \\
${ }^{208} \mathrm{~Pb}\left({ }^{50} \mathrm{Ti}, \mathrm{n}\right)$ & ${ }^{257} \mathrm{Rf}$ & $39.5 \pm 1.2$ & $3.5 \pm 1.0$ & 29.8 & 7.7 & 15.6 \\
\hline
\end{tabular}

${ }^{\mathrm{a}}$ The distribution was only slightly peaked in the horizontal direction.

9 Table 2. RTC conditions used in characterization experiments and simulations. Each column 10 represents a separate experiment except the final column, which represents a simulation 11 discussed in Sec. 7. A series of resistors creates a linear decrease in potential between ring 1 and 12 ring 4 and controls the potentials of rings 2 and 3 . In all experimental cases, $P_{\mathrm{MC}}$ was 228 torr $13(30.4 \mathrm{kPa}), \mathrm{DP}$ was 10 torr $(1.3 \mathrm{kPa})$, and the flow rate was $2.5 \mathrm{~L} / \mathrm{min}$. In the online 14 experiments, the total Mylar degrader thickness was $10.3 \pm 0.2 \mu \mathrm{m}\left(\theta=45^{\circ}\right)$. See Eq. (3) for the 15 definition of $\varepsilon_{\mathrm{RTC}}^{\prime}$.

\begin{tabular}{cccccccc}
\hline Description & \multicolumn{9}{c}{${ }^{118} \mathrm{Sn}\left({ }^{40} \mathrm{Ar}, 6 \mathrm{n}\right)$} & & $\begin{array}{c}{ }^{228} \mathrm{Th} \\
\text { Source }\end{array}$ & $\begin{array}{c}\text { "Mesh" } \\
\text { Proposal }\end{array}$ \\
\hline Nuclide & ${ }^{152} \mathrm{Er}$ & ${ }^{152} \mathrm{Er}$ & ${ }^{152} \mathrm{Er}$ & ${ }^{152} \mathrm{Er}$ & ${ }^{152} \mathrm{Er}$ & ${ }^{216} \mathrm{Po}$ & ${ }^{152} \mathrm{Er}$ \\
\hline Biases & & & & & & & \\
Window (V) & 0 & 11.0 & 21.6 & 42.4 & 84.0 & 420 & 320 \\
Inner Chamber (V) & 0 & 11.0 & 21.6 & 42.4 & 84.0 & 420 & 320 \\
Ring 1 (V) & 0 & 10.8 & 21.6 & 42.4 & 83.8 & 420 & 320 \\
Ring 4 (V) & 0 & 10.4 & 21.6 & 42.4 & 83.8 & 320 & 320 \\
Flower 1 (V) & 0 & 10.0 & 20.6 & 40.0 & 80.0 & 305 & 305 \\
Flower 2(V) & 0 & 7.6 & 15.0 & 30.0 & 60.2 & 250 & 250 \\
Flower 3 (V) & 0 & 4.4 & 9.6 & 19.4 & 39.4 & 200 & 200 \\
Flower 4 (V) & 0 & 2.2 & 4.8 & 9.8 & 19.8 & 150 & 150 \\
Nozzle (V) & 0 & 2.2 & 4.8 & 9.8 & 19.8 & 150 & 150 \\
\hline$\varepsilon_{\text {RTC }}^{\prime}(\%)$ & $25 \pm 3$ & $35 \pm 4$ & $43 \pm 5$ & $39 \pm 5$ & $33 \pm 4$ & $-{ }^{\mathrm{a}}$ & - \\
\hline$\tau_{\mathrm{RTC}}(\mathrm{s})$ & $8.5 \pm 1.0$ & $7.2 \pm 2.4$ & $6.9 \pm 0.6$ & $8.1 \pm 1.9$ & $2.2 \pm 1.2$ & $-{ }^{\mathrm{a}}$ & - \\
\hline
\end{tabular}


1 Table 3. Maximum measured DP and corresponding flow rate as a function of capillary inner 2 diameter (i.d.). In all cases, the MC pressure was 228 torr $(30.4 \mathrm{kPa})$ and the length of the 3 capillary was 25 feet $(7.6 \mathrm{~m})$. Flow rates are equivalent to $\mathrm{He}$ at $25^{\circ} \mathrm{C}$ and $100 \mathrm{kPa}$. A 4 maximum DP of zero indicates that the capillary was too narrow and the conductance was too 5 low to maintain any difference in pressure.

\begin{tabular}{|c|c|c|c|c|}
\hline \multicolumn{2}{|c|}{ Capillary i.d. } & \multicolumn{2}{|c|}{ Max. Measured DP } & $\begin{array}{c}\text { Measured } \\
\text { Flow Rate } \\
\text { (L/min) }\end{array}$ \\
\hline $1 / 16$ & 1.6 & 0 & 0 & $<0.2$ \\
\hline $3 / 32$ & 2.4 & 3.1 & 0.41 & 1.1 \\
\hline $1 / 8$ & 3.2 & 19 & 2.5 & 2.8 \\
\hline $5 / 32$ & 4.0 & 40 & 5.3 & 4.0 \\
\hline
\end{tabular}

7 Table 4. Maximum efficiencies under various conditions. $\varepsilon_{\mathrm{RTC}}$ is the fraction of ions impinging 8 on the RTC window that are detected by the AC detector, $\varepsilon_{\mathrm{RTC}}^{\prime}$ is the efficiency that could 9 theoretically be achieved if no ions stopped in the window, and $\varepsilon_{\mathrm{RTC}}^{\prime \prime}$ is the same as $\varepsilon_{\mathrm{RTC}}^{\prime}$ but 10 corrected for transportation time. See Eqs. (2), (3), and (5) for definitions, respectively. The 11 degrader thicknesses represent the total of both Mylar degraders.

\begin{tabular}{|c|c|c|}
\hline Quantity & $\begin{array}{l}\text { Gas Flow } \\
\text { Only }\end{array}$ & $\begin{array}{l}\text { Gas Flow and } \\
\text { Electric Field }\end{array}$ \\
\hline Maximum $\varepsilon_{\mathrm{RTC}}(\%)$ & $21 \pm 3$ & $35 \pm 4$ \\
\hline $\begin{array}{c}\theta \\
\text { Degrader }(\mu \mathrm{m})\end{array}$ & $\begin{array}{c}42.5^{\circ} \\
10.0 \pm 0.2\end{array}$ & $\begin{array}{c}42.5^{\circ} \\
10.0 \pm 0.2\end{array}$ \\
\hline Maximum $\varepsilon_{\mathrm{RTC}}^{\prime}(\%)$ & $25 \pm 3$ & $44 \pm 5^{\mathrm{a}}$ \\
\hline $\begin{array}{c}\theta \\
\text { Degrader }(\mu \mathrm{m})\end{array}$ & $\begin{array}{c}45^{\circ} \\
10.3 \pm 0.2\end{array}$ & $\begin{array}{c}48^{\circ} \\
10.8 \pm 0.2\end{array}$ \\
\hline Maximum $\varepsilon_{\mathrm{RTC}}^{\prime \prime}(\%)$ & $44 \pm 6$ & $70 \pm 9$ \\
\hline $\begin{array}{c}\theta \\
\text { Degrader (um) }\end{array}$ & $\begin{array}{c}45^{\circ} \\
10.3 \pm 0.2\end{array}$ & $\begin{array}{c}48^{\circ} \\
10.8 \pm 0.2\end{array}$ \\
\hline is datum differs & $10.0 \pm 0.2$ & in Table \\
\hline
\end{tabular}




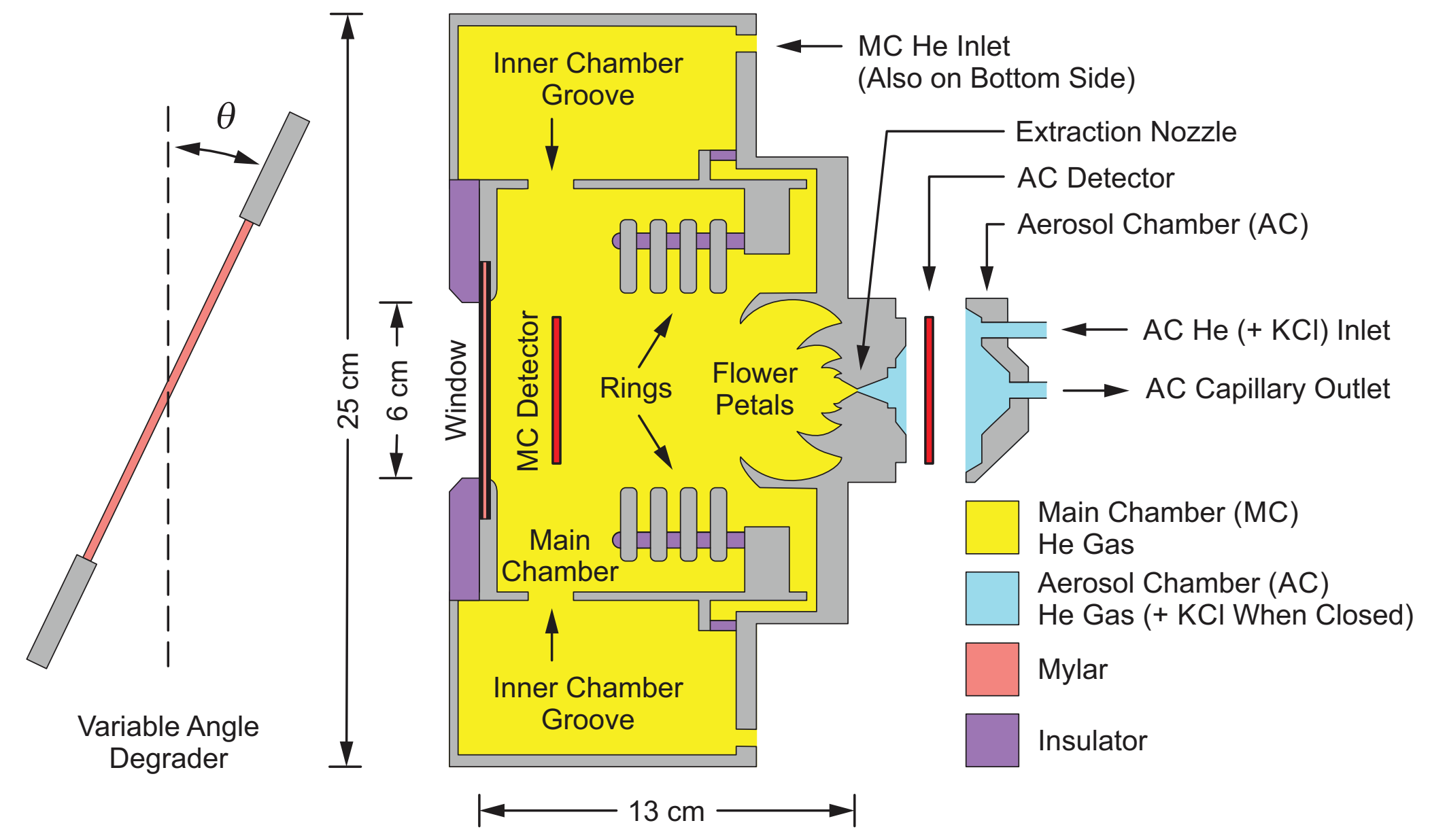




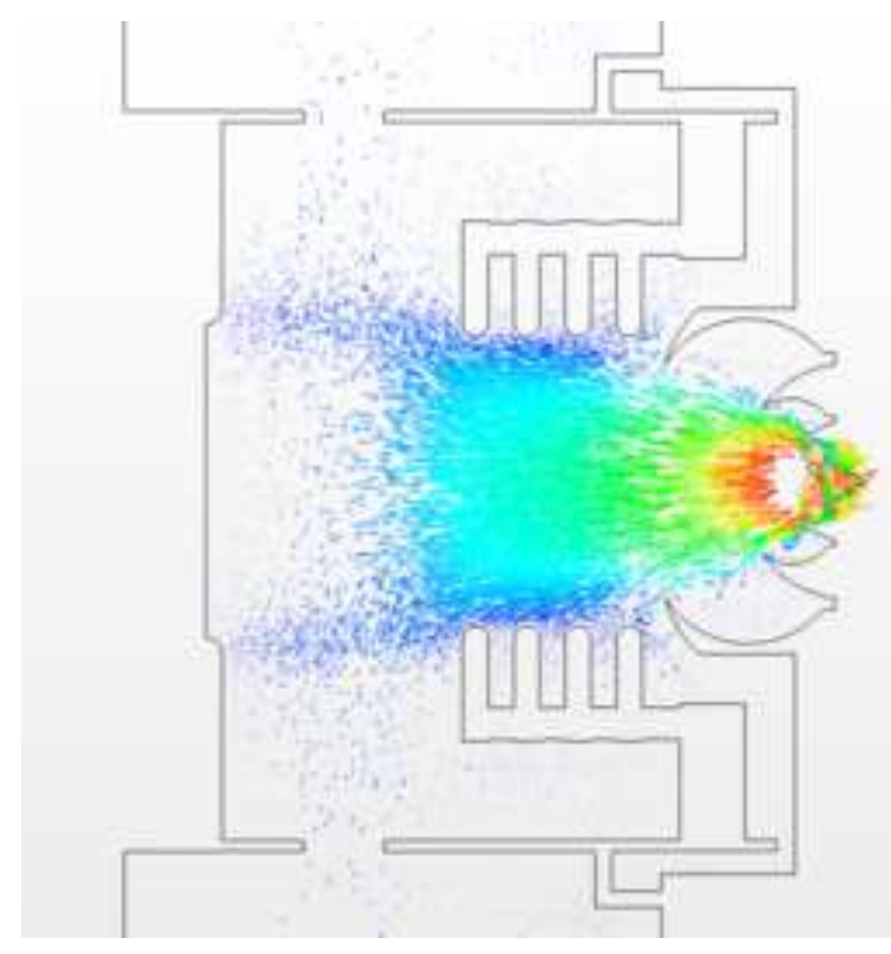

(a)

Velocity ( $\mathrm{m} / \mathrm{s})$

020

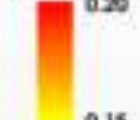

0.15

Q.10

0.05

ow

(b)

Velocity $(\mathrm{m} / \mathrm{s})$

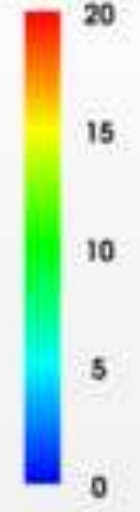

.

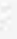

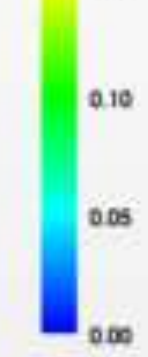



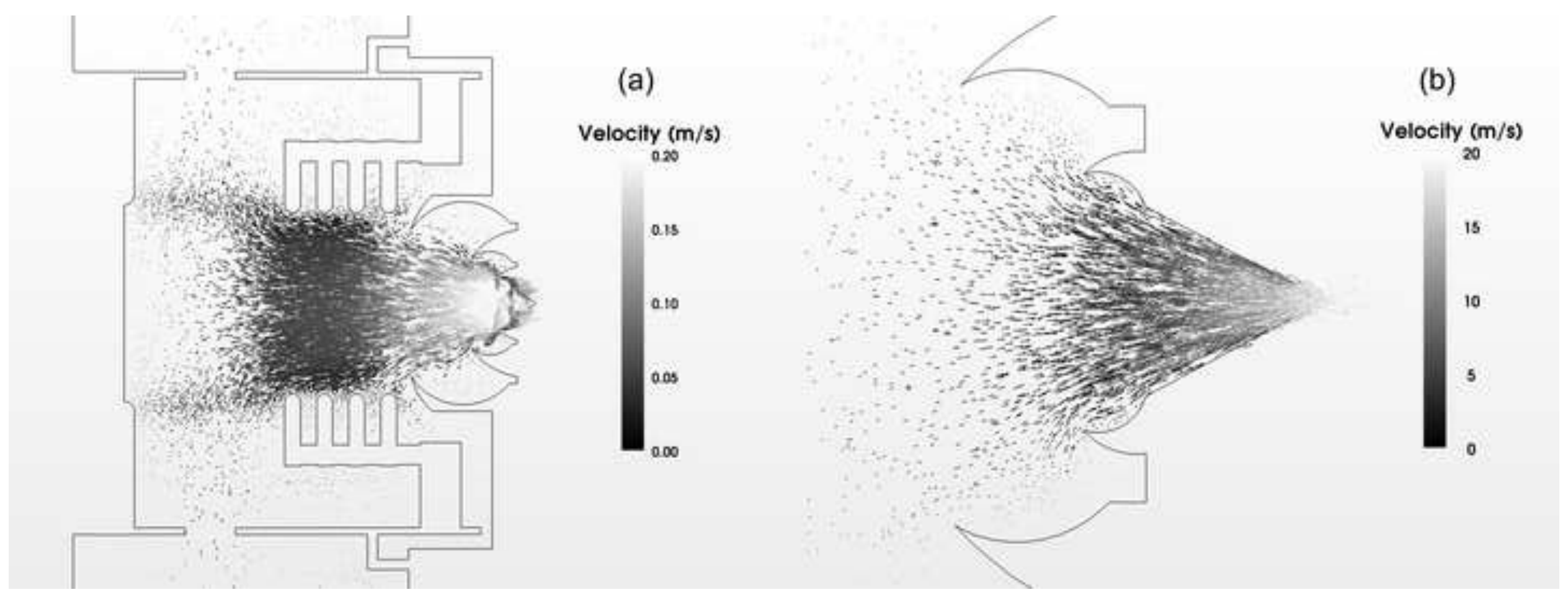

(b) locity $(\mathrm{m} / \mathrm{s})$

15

10

5
0 
Figure 3
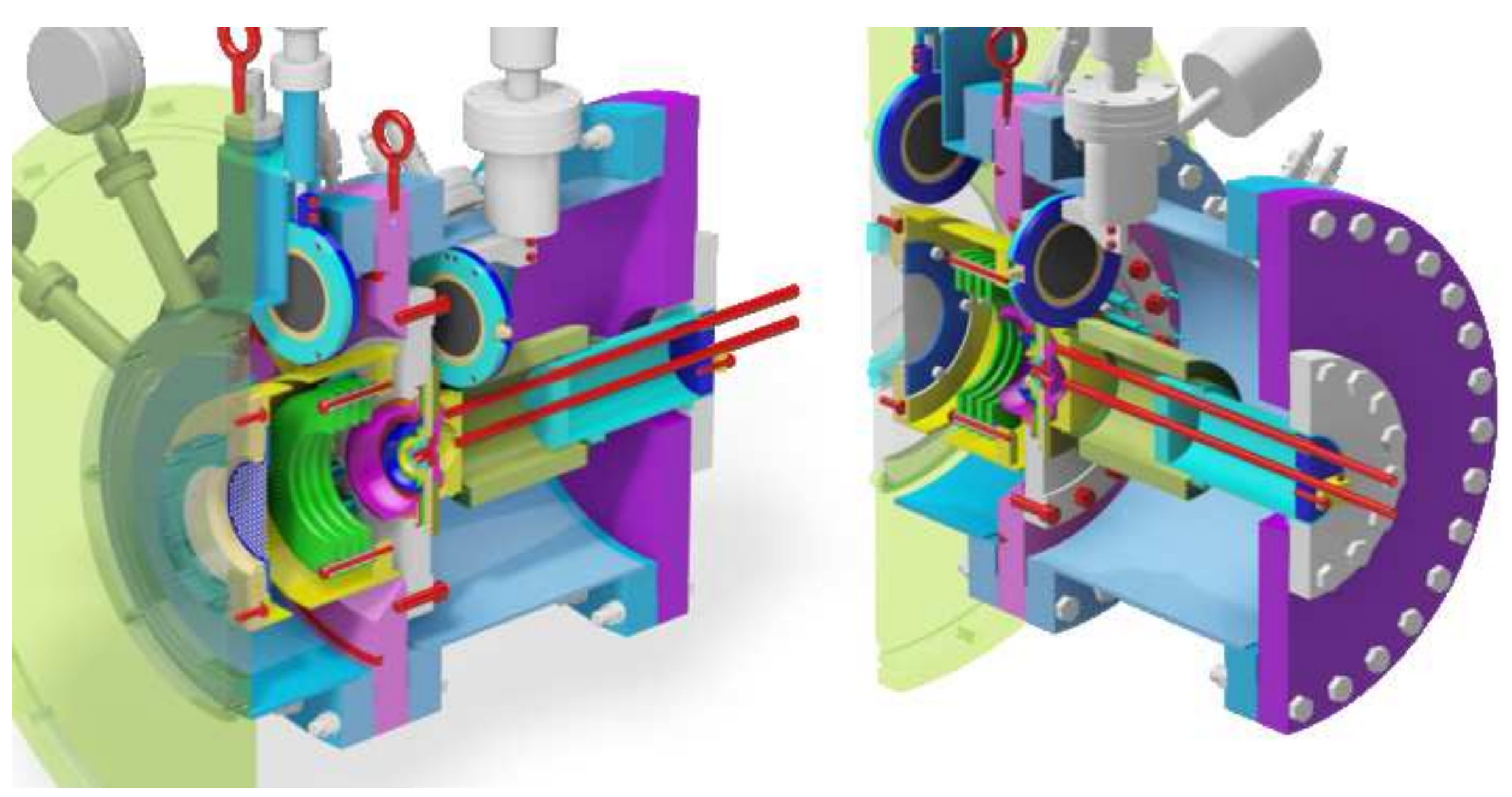


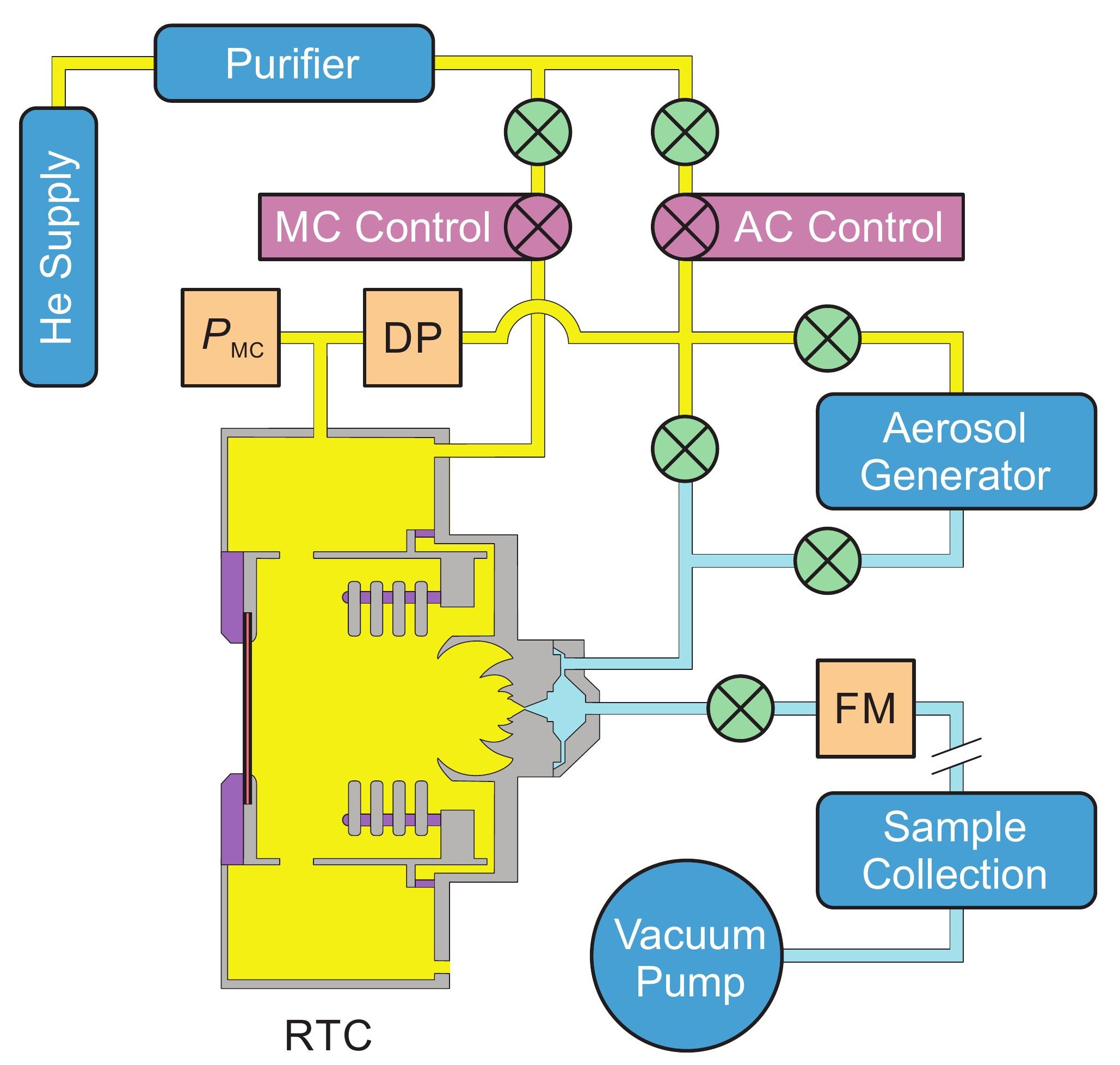

Figure 4 
Figure 5

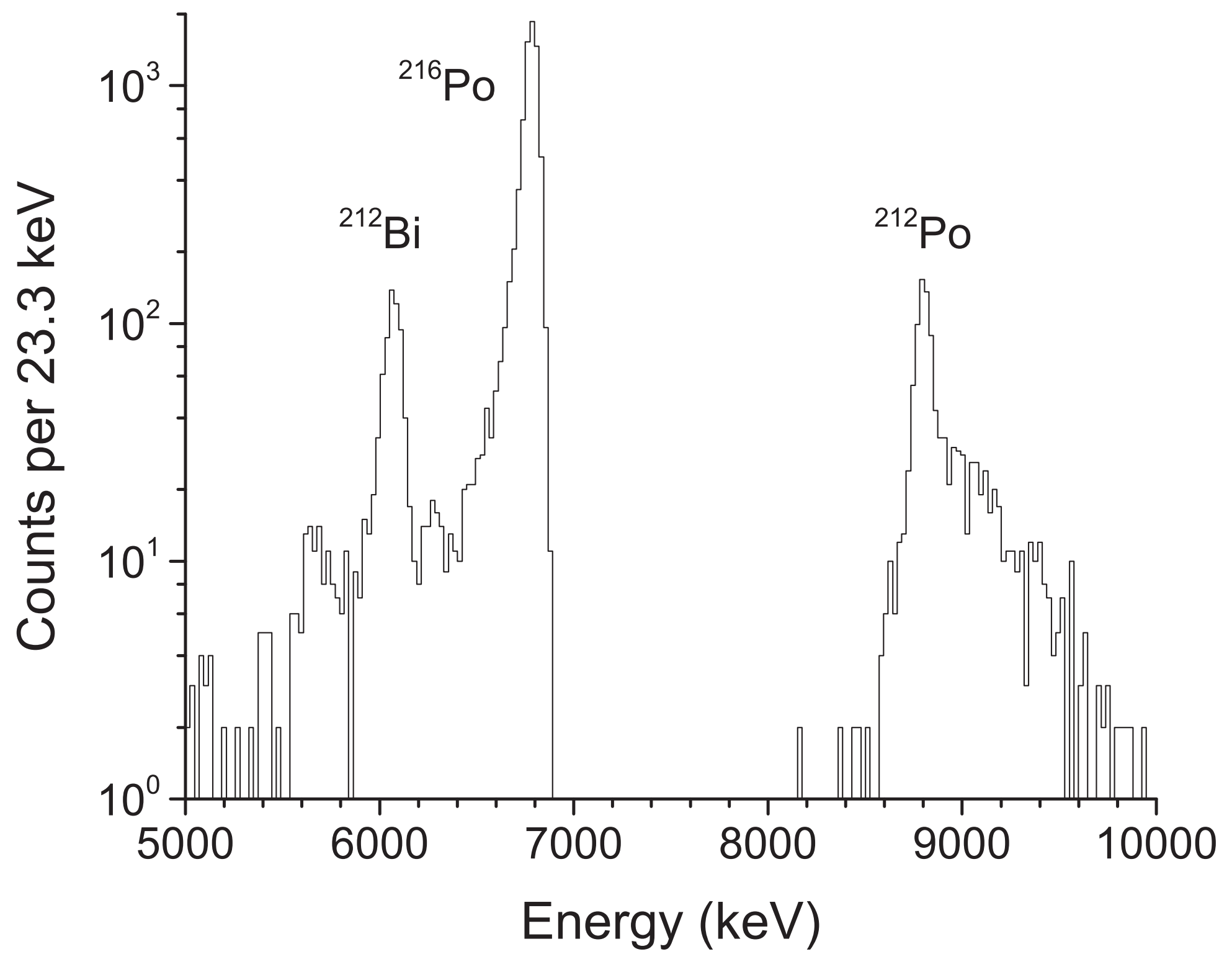



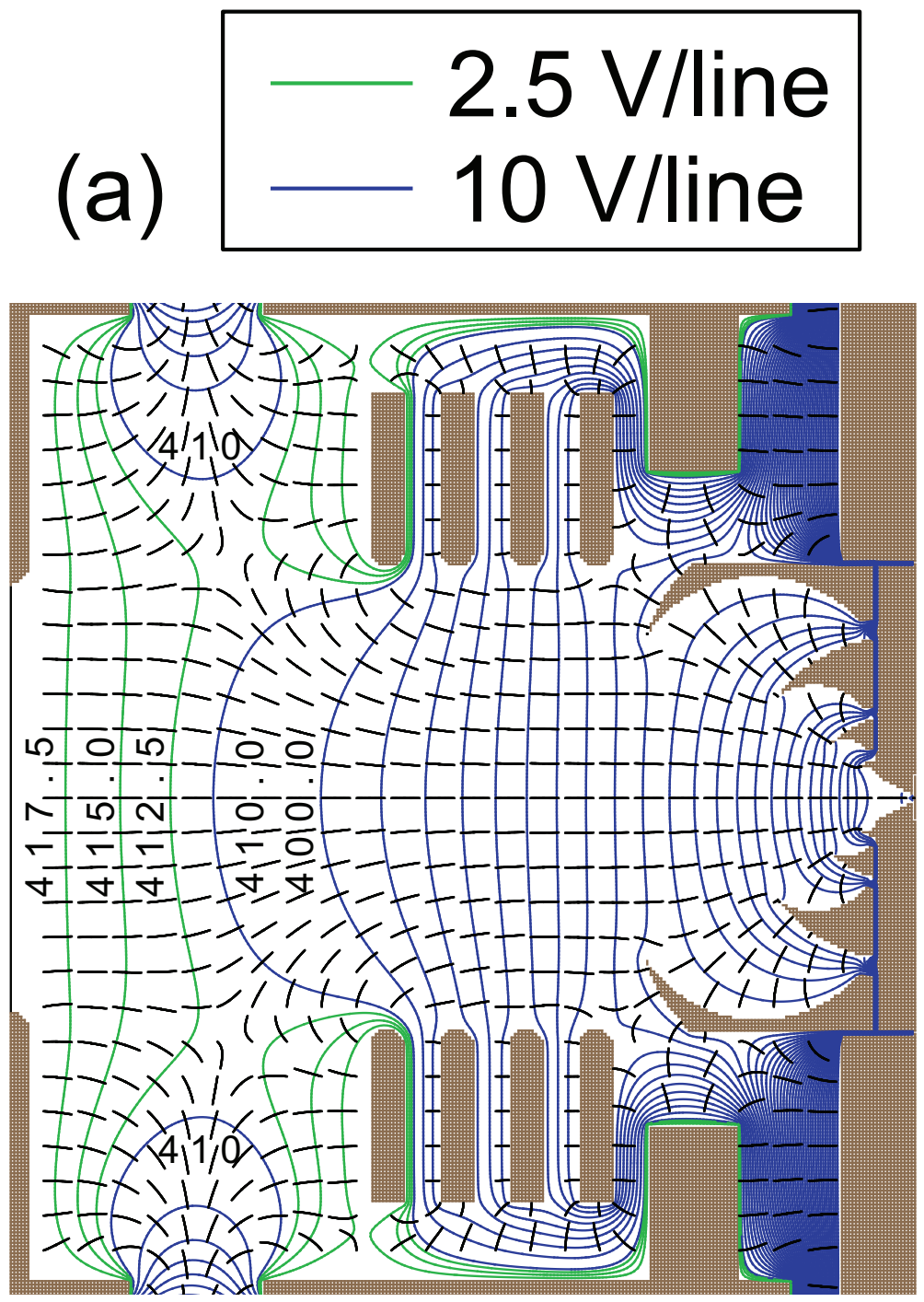
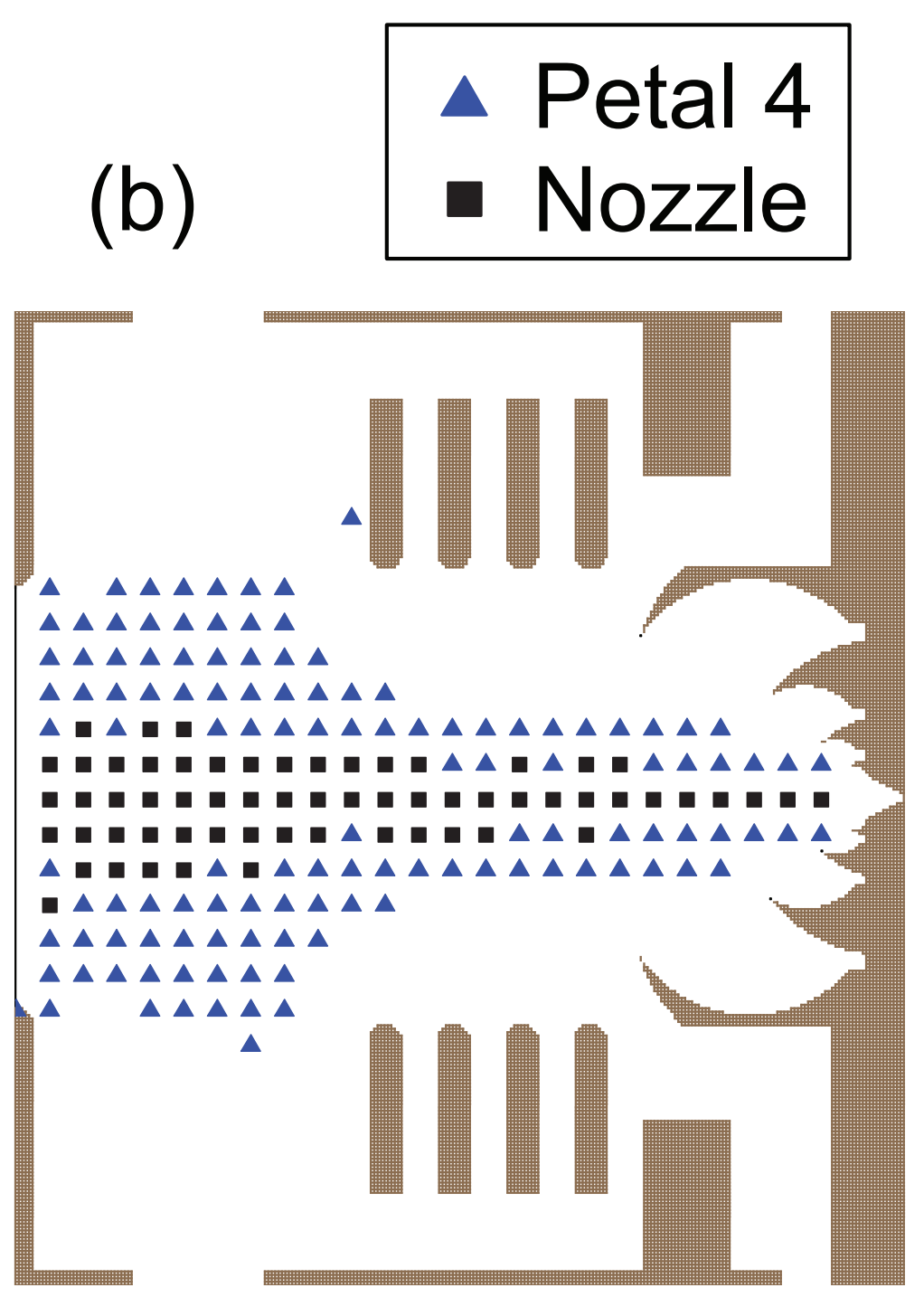


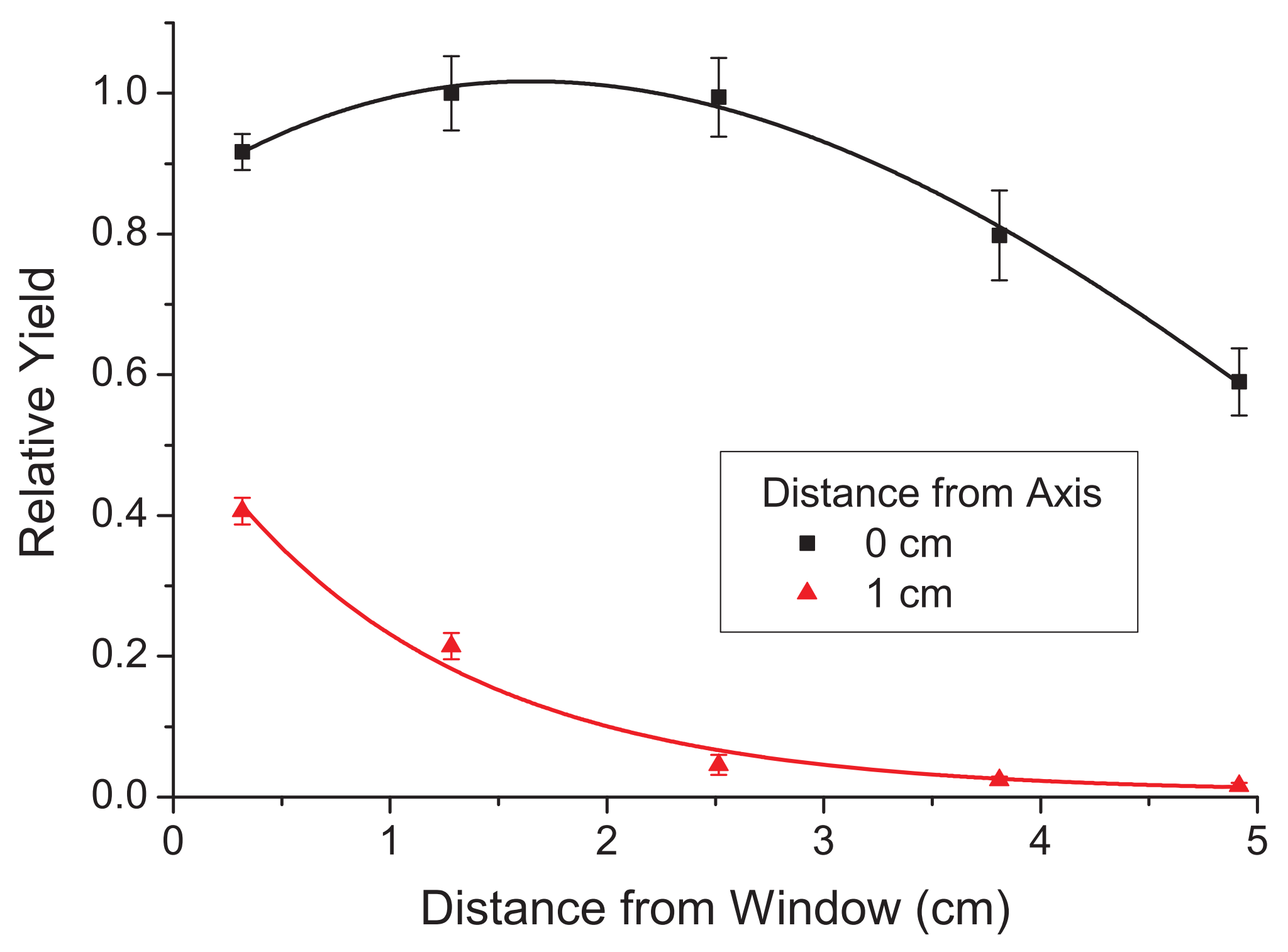

Figure 7 
Figure 8
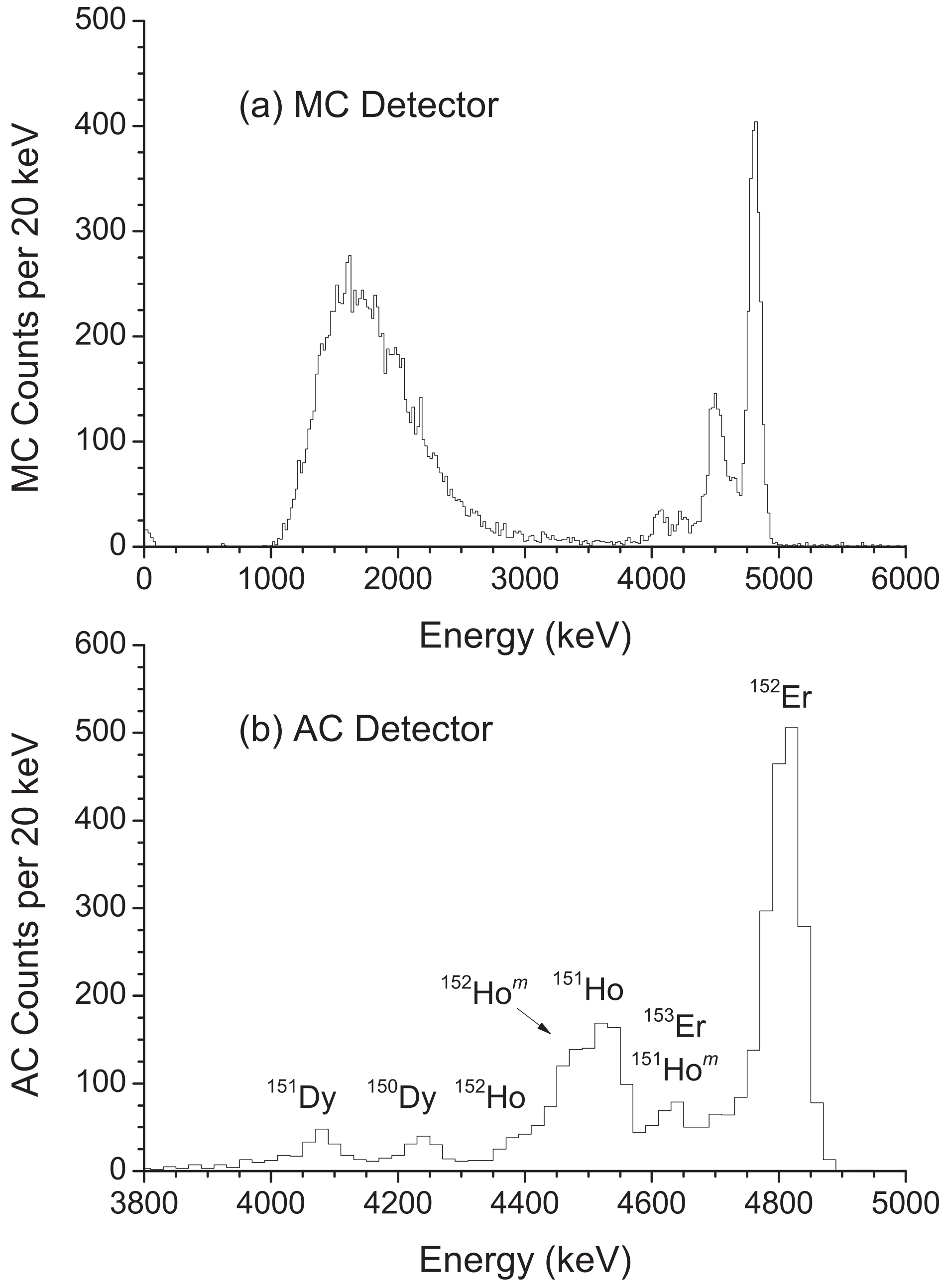
$\theta$ (degrees)

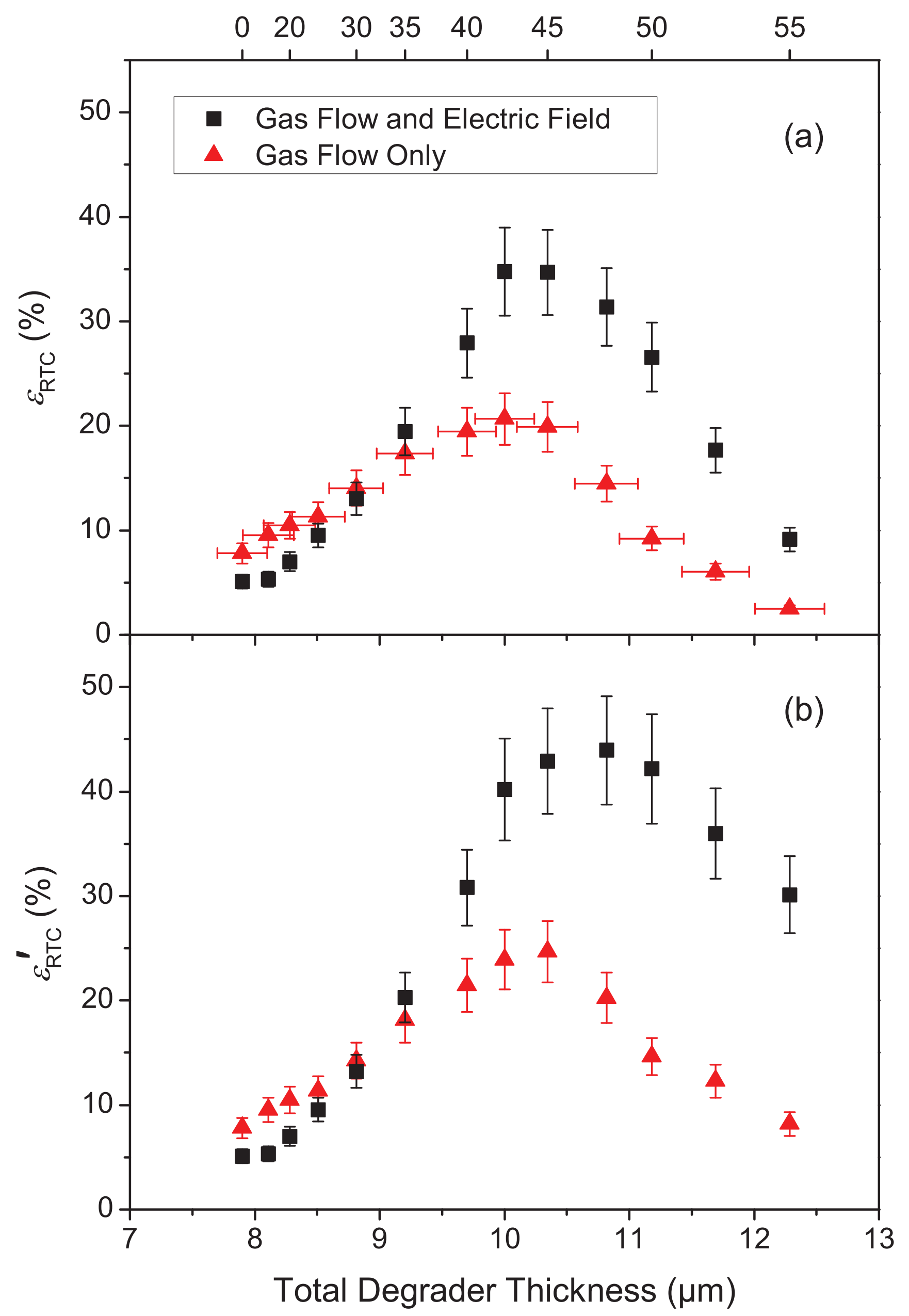




\section{(a) $-0.5 \mathrm{~V} /$ line}

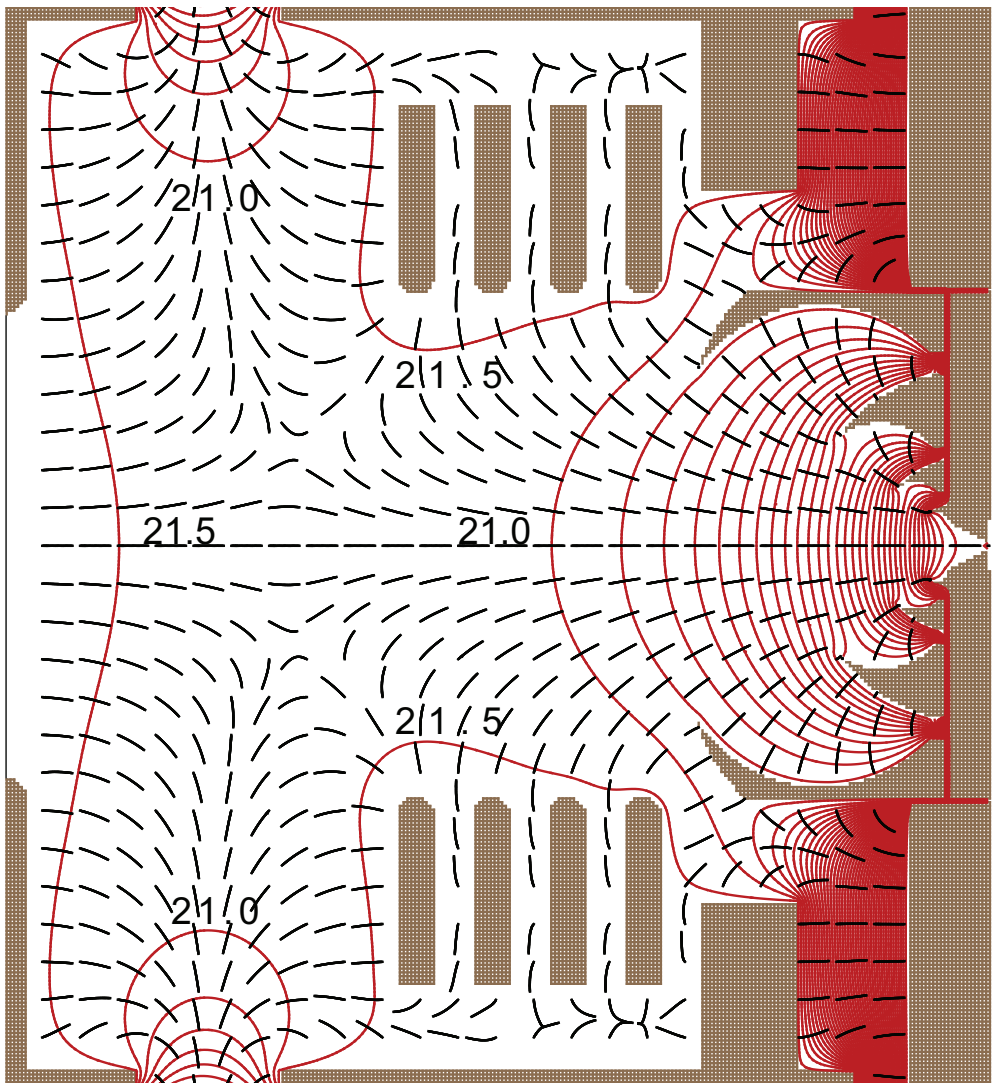

\section{- Petal 4 - Nozzle}

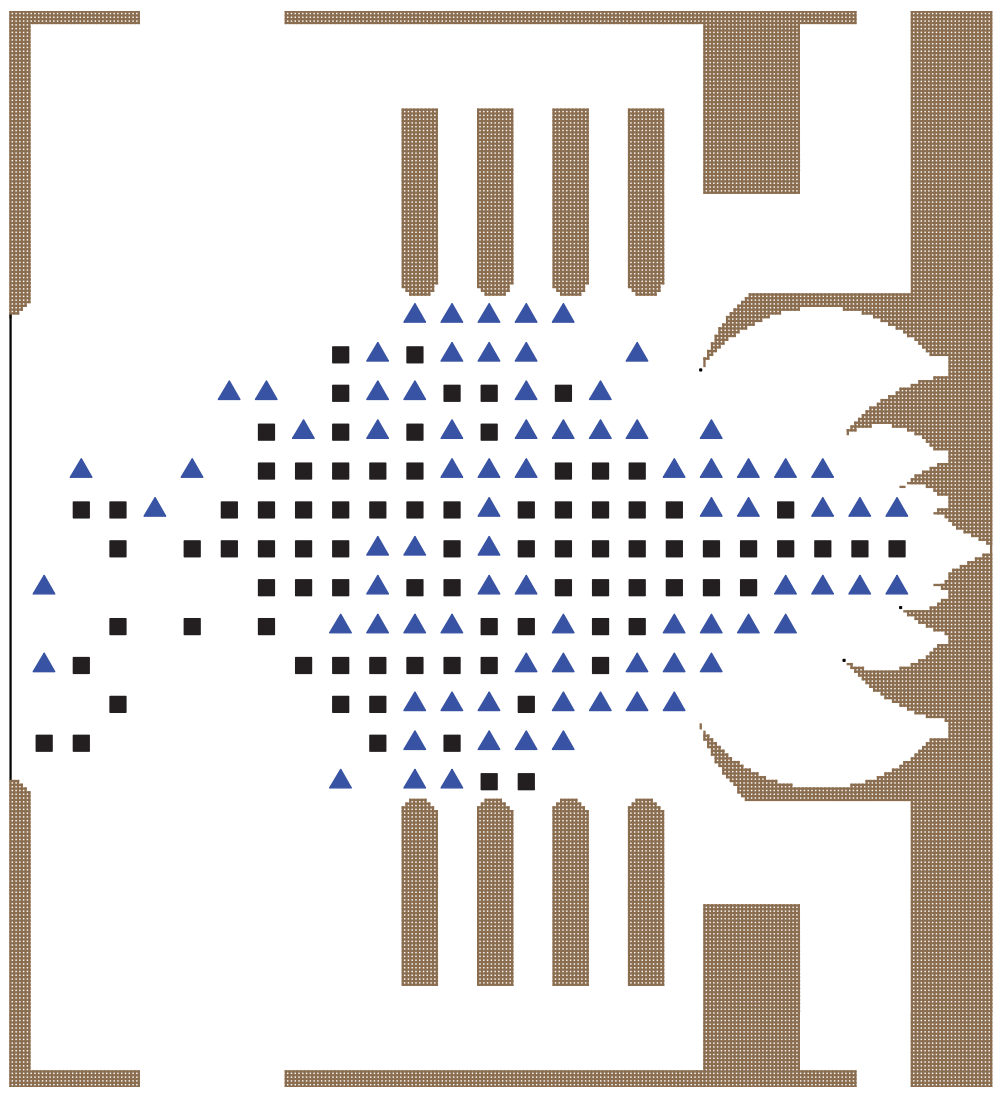



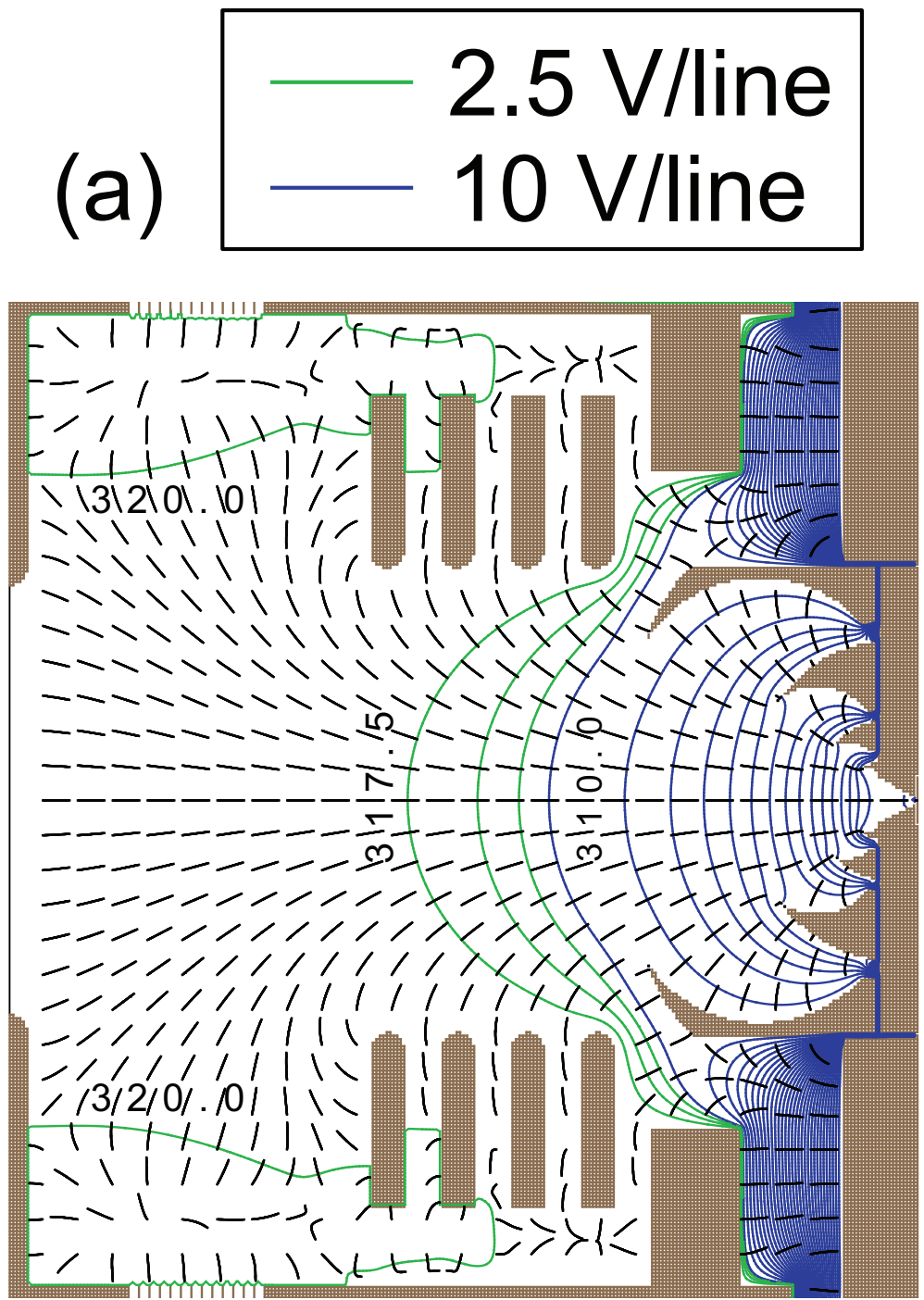

(b)

\section{- Petal 4 \\ - Nozzle}

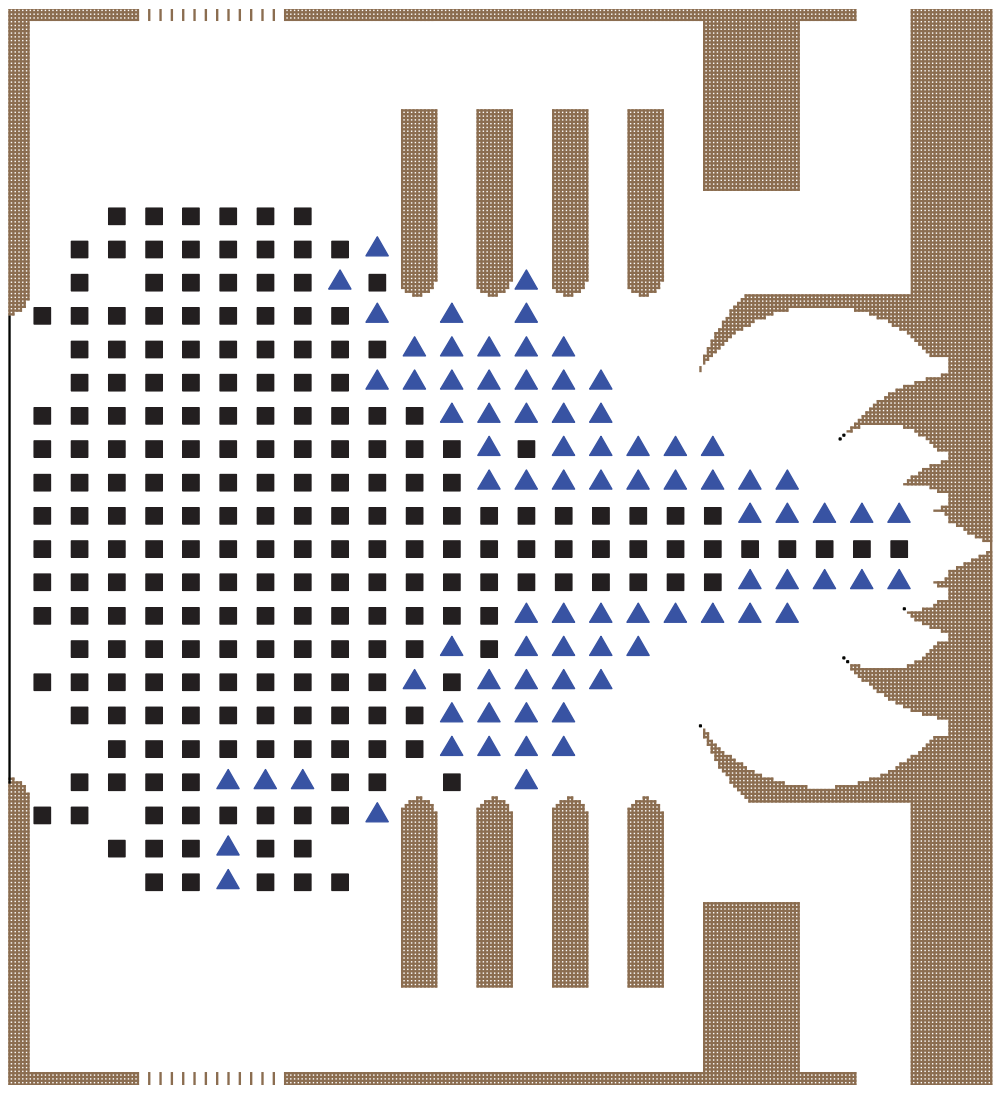

